ARTICLE

\title{
Immunomodulating nano-adaptors potentiate antibody-based cancer immunotherapy
}

\author{
Cheng-Tao Jiang (1) ${ }^{1,8}$, Kai-Ge Chen ${ }^{2,8}$, An Liu² ${ }^{2}$ Hua Huang ${ }^{1}$, Ya-Nan Fan ${ }^{1}$, Dong-Kun Zhao', Qian-Ni Ye ${ }^{1}$, \\ Hou-Bing Zhang ${ }^{1}$, Cong-Fei Xu1,3, Song Shen (1) ${ }^{1,4,5 凶}$, Meng-Hua Xiong ${ }^{1}$, Jin-Zhi Du ${ }^{1}$, Xian-Zhu Yang ${ }^{1,5} \&$ \\ Jun Wang (iD $1,5,6,7 凶$
}

Modulating effector immune cells via monoclonal antibodies (mAbs) and facilitating the coengagement of $T$ cells and tumor cells via chimeric antigen receptor- $T$ cells or bispecific $T$ cell-engaging antibodies are two typical cancer immunotherapy approaches. We speculated that immobilizing two types of mAbs against effector cells and tumor cells on a single nanoparticle could integrate the functions of these two approaches, as the engineered formulation (immunomodulating nano-adaptor, imNA) could potentially associate with both cells and bridge them together like an 'adaptor' while maintaining the immunomodulatory properties of the parental mAbs. However, existing mAbs-immobilization strategies mainly rely on a chemical reaction, a process that is rough and difficult to control. Here, we build up a versatile antibody immobilization platform by conjugating anti-lgG (Fc specific) antibody $(\alpha F c)$ onto the nanoparticle surface ( $\alpha \mathrm{Fc}-\mathrm{NP})$, and confirm that $\alpha \mathrm{Fc}-\mathrm{NP}$ could conveniently and efficiently immobilize two types of mAbs through Fc-specific noncovalent interactions to form imNAs. Finally, we validate the superiority of imNAs over the mixture of parental mAbs in $T$ cell-, natural killer cell- and macrophage-mediated antitumor immune responses in multiple murine tumor models.

\footnotetext{
${ }^{1}$ School of Biomedical Sciences and Engineering, South China University of Technology, Guangzhou International Campus, Guangzhou 511442, PR China. ${ }^{2}$ School of Life Sciences, University of Science and Technology of China, Hefei 230027, PR China. ${ }^{3}$ Shenzhen Bay Laboratory, Shenzhen 518132, PR China. ${ }^{4}$ National Engineering Research Center for Tissue Restoration and Reconstruction, South China University of Technology, Guangzhou 510006, PR China. ${ }^{5}$ Key Laboratory of Biomedical Engineering of Guangdong Province, and Innovation Center for Tissue Restoration and Reconstruction, South China University of Technology, Guangzhou 510006, PR China. ${ }^{6}$ Bioland Laboratory (Guangzhou Regenerative Medicine and Health Guangdong Laboratory), Guangzhou 510005, PR China. ${ }^{7}$ Key Laboratory of Biomedical Materials and Engineering of the Ministry of Education, South China University of Technology, Guangzhou 510006, PR China. ${ }^{8}$ These authors contributed equally: Cheng-Tao Jiang, Kai-Ge Chen. ${ }^{凶}$ email: shensong@scut.edu.cn; mcjwang@scut.edu.cn
} 
C urrent strategies to boost cancer immunotherapy in the clinic include two broad categories. One category is to utilize immunomodulatory monoclonal antibodies (mAbs) to reinvigorate dysfunctional $\mathrm{T}$ lymphocytes ${ }^{1,2}$. mAbs that block cytotoxic T-lymphocyte-associated protein 4 (CTLA4), programmed cell death protein 1 (PD1), and programmed cell death ligand 1 (PDL1) represent the predominant modalities for cancer immunotherapy and have revolutionized the cancer treatment paradigm in recent years ${ }^{3,4}$. Of note, numerous mAbs capable of potentiating the antitumor activities of innate immune cells (for example, natural killer cells and macrophages) are also under intense investigation ${ }^{5-7}$.

Parallel to the immunomodulatory mAbs, the other strategy is to facilitate the engagement of effector immune cells and tumor cells via chimeric antigen receptor (CAR) $\mathrm{T}$ cells or bispecific $\mathrm{T}$ cell-engaging antibodies (BiTEs) ${ }^{8-10}$. The former are engineered autologous $\mathrm{T}$ cells expressing an artificial receptor that recognizes a specific tumor-associated antigen (TAA) ${ }^{11,12}$, and the latter is an advanced format of bispecific antibodies (bsAbs) that comprise two-variable fragments capable of simultaneously binding to the $\mathrm{T}$ cell receptor complex and $\mathrm{TAA}^{13-15}$. Although the anticancer mechanisms are different, CAR T cells and BiTE share a common feature of triggering physical connections between effector cells and tumor cells and facilitating cytotoxicity against the latter $9,16,17$.

To fully exploit the advantages of these two strategies, the combination of immunomodulatory mAbs and CAR $\mathrm{T}$ cells or BiTEs have been combined and have shown improved efficacy in comparison with individual approaches alone; ${ }^{18-20}$ however, the two therapeutics in most cases work independently. We speculated that integrating the features of these two strategies into one system may further boost immunotherapy. We proposed that nanoparticle immobilizing two types of mAbs targeting effector cells and tumor cells could be such a system, as the engineered formulation potentially has two distinctive features and acts as an immunomodulating nano-adaptors (imNAs). First, the immobilized parental $\mathrm{mAbs}$ maintain their immunomodulatory properties, and second, because of their multivalence, they have a strong affinity for antigens on both cells and can physically bridge them together like an 'adaptor'21. It is reasonable to predict that imNAs could achieve amplified and unachievable antitumor effects over a mixture of two types of mAbs. For the immobilization of mAbs onto nanoparticles, previously reported approaches mainly rely on chemical reactions; ${ }^{21,22}$ however, this process is difficult to control due to the high molecular weight of mAbs and nanoparticles, and it may also hurt the valency of mAbs, limiting their clinical translation ${ }^{23}$.

In this work, considering that $\mathrm{Fc}$ regions are identical or conserved in all IgG antibodies and that an anti-IgG (Fc specific) antibody $(\mathrm{aFc})$ can specifically recognize and bind any mAbs comprising the Fc fragment through noncovalent interactions ${ }^{24,25}$, we propose to build up a versatile antibody immobilization platform by conjugating $\alpha \mathrm{Fc}$ onto the nanoparticle surface ( $\alpha \mathrm{Fc}-\mathrm{NP}$ ) (Fig. 1a). We confirm that $\alpha \mathrm{Fc}-\mathrm{NP}$ could conveniently and efficiently immobilize two types of mAbs after gentle mixing to form imNAs without compromising the antigen-binding capabilities of the parental mAbs (Fig. 1b). We further select the immune checkpoint inhibitors aPD1 (an antiPD1 antibody) and aPDL1 (an anti-PDL1 antibody) as model mAbs and show that imNA $\mathrm{APD}_{\text {\& }}$ aPDL1 could effectively promote $\mathrm{T}$ cell/tumor cell interactions and strikingly augment $\mathrm{T}$ cellmediated antitumor immunity in vitro and in vivo, in compared with the combination of soluble or nanoparticle-immobilized aPD1 and aPDL1. Notably, the superiority of imNAs over the mixture of parental mAbs is validated in natural killer cell- and macrophage-mediated antitumor immune responses in multiple murine tumor models (Fig. 1c). Collectively, we provide a convenient and well-controlled methodology for the engineering of imNAs, and the simplicity, versatility, and effectiveness of the methodology make imNAs an attractive candidate for clinical translation.

\section{Results}

Design and characterization of the antibody immobilization platform. To fabricate imNAs conveniently and efficiently, we proposed to build up a versatile antibody immobilization platform by conjugating anti-IgG (Fc specific) antibody $(\alpha \mathrm{Fc})$ onto the surface of nanoparticles (NP). Since the Fc regions are identical in all IgG antibodies from the same host species and are well-conserved between different host species $^{26}$, it is reasonable to predict that $\alpha \mathrm{Fc}$-conjugated nanoparticles ( $\alpha \mathrm{Fc}-\mathrm{NP})$ could recognize and immobilize any $\mathrm{mAb}$ containing the $\mathrm{Fc}$ fragment. To maximize the mAb-binding capability of $a \mathrm{Fc}$, an oriented conjugation approach was employed to engineer aFc-NP (Fig. 2a): the hydroxyl groups in carbohydrate residues, which are typically located in the heavy chain $\mathrm{CH} 2$ domain of $\mathrm{aFc}^{27}$, were first oxidized to aldehyde groups by sodium periodate (Supplementary Fig. 1), followed by a condensation reaction with aminated polystyrene NP and reductive amination of Schiff bases with sodium borohydride (Fig. 2a). Over $80 \%$ of $\alpha \mathrm{Fc}$ was immobilized onto NP when the mass ratio of NP to aFc was equal to or greater than 5:1, as determined by enzyme-linked immunosorbent assay (ELISA) (Fig. 2b) and ultra-performance liquid chromatography (UPLC) (Supplementary Fig. 2). Considering the aFc-binding efficacy and $\alpha \mathrm{Fc}$-loading content, a NP: $\alpha \mathrm{Fc}$ ratio of $5: 1$ was selected for the construction of $\alpha \mathrm{Fc}-\mathrm{NP}$ in the subsequent investigation, and $1 \mathrm{mg}$ NP could bind approximately $160 \mu \mathrm{g} \alpha \mathrm{Fc}$ at this ratio, as determined by ELISA.

As measured by dynamic light scattering (DLS), the average hydrodynamic diameter of $\mathrm{aFc}-\mathrm{NP}$ was $\sim 152.8 \mathrm{~nm}$, approximately $30 \mathrm{~nm}$ larger than that of polystyrene NP (Fig. 2c), while the typical size of an antibody molecule is $10-15 \mathrm{~nm}^{28}$. Scanning electron microscopy (SEM) images confirmed that both NP and $\alpha \mathrm{Fc}-\mathrm{NP}$ were homogeneously spherical; intriguingly, the former had a smooth surface while the latter had a rough surface (Fig. 2d). Collectively, compared with naked polystyrene NP, aFcNP showed remarkable changes in terms of size distribution and morphology, indicating that a layer of antibodies was attached to the surface of the NP. To further substantiate that $\mathrm{aFc}$ was oriented chemically conjugated but not physically adsorbed onto $\mathrm{NP}$, the heavy chains (HC) and light chains (LC) of soluble $\alpha \mathrm{Fc}$ and $\alpha \mathrm{Fc}-\mathrm{NP}$ were separated by $\beta$-mercaptoethanol, which can break the interchain disulfide bonds, followed by gradient SDSPAGE (sodium dodecyl sulfate-polyacrylamide gel electrophoresis) and Coomassie Blue staining (Supplementary Fig. 3). As shown in Fig. 2e, in comparison with free $\alpha \mathrm{Fc}$ counterpart, the $\mathrm{HC}$ band $(\sim 50 \mathrm{kDa})$ was substantially dimmer in the aFc-NP group when the LC bands $(\sim 25 \mathrm{kDa})$ were identical; the missing HC was thought to be linked to the NP through carbohydrates and blocked in the sample well during SDS-PAGE process, confirming that $\mathrm{aFc}$ was chemically conjugated.

Having validated the successful construction of aFc-NP, we further examined whether $\alpha \mathrm{Fc}-\mathrm{NP}$ could serve as a versatile platform for the immobilization of mAbs containing Fc fragments (Fig. 1c). DLS measurements showed that the average hydrodynamic diameter of $\alpha \mathrm{Fc}-\mathrm{NP}$ increased by approximately $30 \mathrm{~nm}$ after incubation with $\alpha \mathrm{PD} 1$ at $4{ }^{\circ} \mathrm{C}$ for $2 \mathrm{~h}$, indicating the immobilization of $\alpha \mathrm{PD} 1$ to $\alpha \mathrm{Fc}-\mathrm{NP}\left(\alpha \mathrm{Fc}-\mathrm{NP}_{\alpha \mathrm{PD} 1}\right)$ (Fig. $\left.2 \mathrm{f}\right)$. To verify that $\alpha \mathrm{Fc}-\mathrm{NP}_{\alpha \mathrm{PD} 1}$ was generated through the specific interaction between $\alpha F c$ and the $F c$ fragment of $\alpha \mathrm{PD} 1, \alpha \mathrm{Fc}$ or an IgG control antibody (anti-trinitrophenol antibody) was 
a
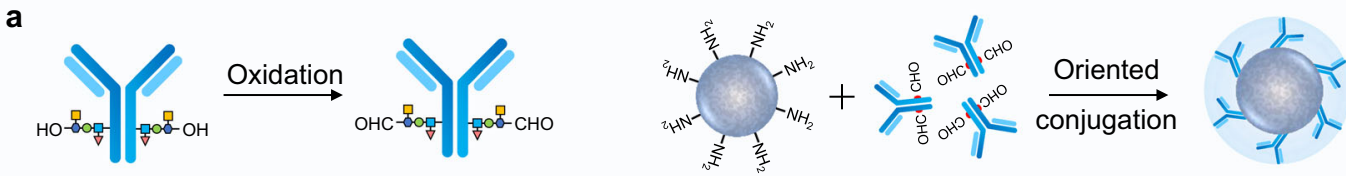

anti-lgG (Fc specific)

Oxidized $\alpha \mathrm{Fc}$

Aminated NP

Oxidized $\alpha \mathrm{Fc}$

mAbs immobilization platform ( $\alpha$ Fc-NP)

b

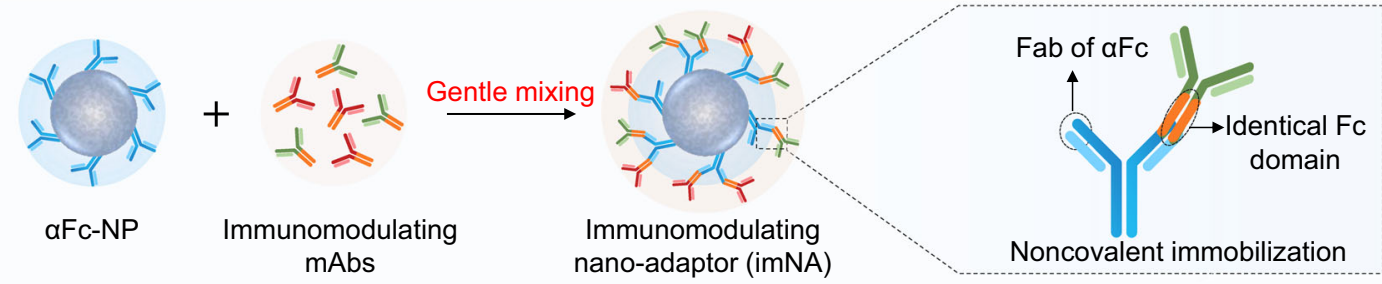

C
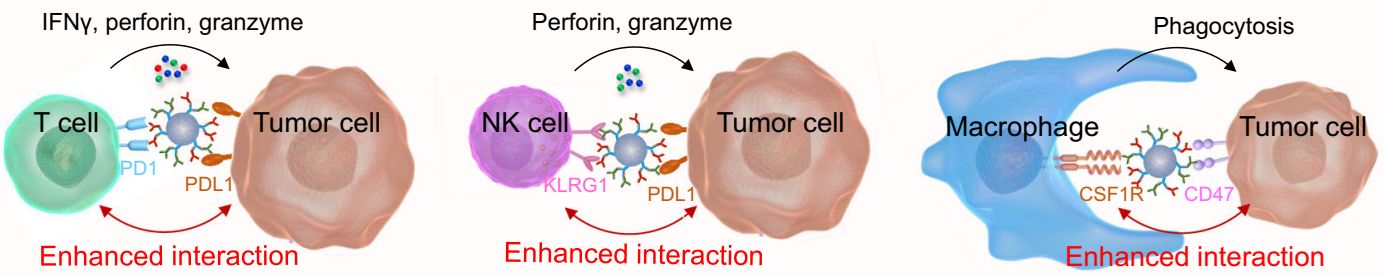

旅 imNA $_{\alpha P D 1}$ aPDL1
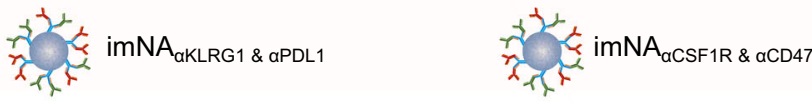

Fig. 1 Schematic illustrating the design of imNAs and their potential to improve antibody-based cancer immunotherapy. a An anti-lgG (Fc specific) antibody $(\alpha \mathrm{Fc}$ ) was oxidized and oriented conjugated onto the surface of nanoparticle to form an antibody immobilization platform ( $\alpha \mathrm{Fc}-\mathrm{NP}$ ). $\mathbf{b}$ Two types of immunomodulating monoclonal antibodies (mAbs) that target effector cells and tumor cells could be immobilized onto $\alpha \mathrm{Fc}-\mathrm{NP}$ after gentle mixing via Fc recognition. The constructed formulations could serve as immunomodulating nano-adaptors (imNAs) as they potentially associate with effector cells and tumor cells simultaneously and bridge them together like an 'adaptor' while maintaining the immunomodulatory properties of the parental mAbs. c The versatility of $\alpha \mathrm{Fc}-\mathrm{NP}$ and superiority of imNAs were validated in T cell-, natural killer cell- and macrophage-mediated antitumor immune responses in multiple murine tumor models.

conjugated to an Alexa Fluor ${ }^{\circledR} 750$ (AF750)-labeled NP and then incubated with Alexa Fluor ${ }^{\circledR} 647$ (AF647)-labeled aPD1, followed by imaging of stochastic optical reconstruction microscopy (STORM) imaging. As shown in Fig. $2 \mathrm{~g}$, green fluorescence (AF647) was rarely observed surrounding NP in the IgG-NP ${ }_{\alpha \mathrm{PD} 1}$ group (upper), while larger formulation and colocalization of two kinds of fluorescence (green and red) were observed in the $\mathrm{aFc}$ $\mathrm{NP}_{\alpha \mathrm{PD} 1}$ group (lower), indicating the integration of $\alpha \mathrm{Fc}-\mathrm{NP}$ and $\alpha \mathrm{PD} 1$. Additionally, $\alpha \mathrm{Fc}-\mathrm{NP}_{\alpha \mathrm{PD} 1}$ remained stable within two days in $5 \%$ glucose solution as measured by the variation of the size distribution (Supplementary Fig. 4). More importantly, when incubated with IgG antibodies from the same or other species, few $\alpha \mathrm{Fc}-\mathrm{NP}$-integrated $\mathrm{\alpha PD} 1$ was rarely replaced by surrounding antibodies (Supplementary Fig. 5), even though the concentration of surrounding antibodies was much higher than aPD1 (Supplementary Fig. 6), indicating that the interaction between $\mathrm{Fc}$ fragments and $\mathrm{aFc}$ was relatively stable.

Next, to examine whether aFc-NP can immobilize more than one type of $\mathrm{mAb}, \alpha \mathrm{Fc}-\mathrm{NP}$ was incubated with FITC-labeled $\alpha \mathrm{PD} 1$ and PerCP-Cy5.5-labeled aPDL1 separately or in combination and then subjected to nanoflow cytometry. The emergence of $\mathrm{FITC}^{+}$PerCP-Cy5.5 $5^{+}$formulations in the combination group confirmed that $a \mathrm{Fc}-\mathrm{NP}$ could simultaneously immobilize two types of mAbs, and it could therefore be a universal platform for antibody-based combination therapy (Fig. 2h). Additionally, after gentle mixing and a short incubation (less than $4 \mathrm{~h}$ ), more than
$80 \%$ of $\alpha \mathrm{PD} 1$ and $\alpha \mathrm{PDL} 1$ was incorporated onto $\alpha \mathrm{Fc}-\mathrm{NP}$ at the $\alpha F c$ : $\alpha$ PD1: $\alpha$ PDL1 ratio of 1:0.5:0.5 (Fig. $2 \mathrm{i}$ ).

As the amount and distribution of antigens on effector cells and tumor cells were different, it was necessary to consider the ratio of the two types of mAbs when used in combination. Intriguingly, we could achieve predetermined ratios of aPD1/ aPDL1 by modifying their feeding amounts (Fig. 2j), confirming that $\alpha \mathrm{Fc}-\mathrm{NP}$ can immobilize mAbs in a well-controlled manner. As random covalent immobilization can reduce the antibody's affinity $^{29,30}$, it is reasonable to speculate that the elaborate strategy we developed could maximally preserve the function of the parental mAbs, which were immobilized through noncovalent interactions but not chemical conjugation. To test this hypothesis, we incubated free $\alpha \mathrm{PD} 1$ or $\alpha \mathrm{Fc}-\mathrm{NP}_{\alpha \mathrm{PD} 1}$ with the recombinant murine PD1 protein and found that attachment to aFc-NP did not affect aPD1's ability to bind the antigen compared with its free counterpart, as demonstrated by similar dissociation constants $\left(\mathrm{K}_{\mathrm{D}}\right)$ (Fig. $\left.2 \mathrm{k}\right)$. $\alpha \mathrm{Fc}-\mathrm{NP}_{\alpha \mathrm{PDL} 1}$ and free $\alpha \mathrm{PDL} 1$ also exhibited similar antigen-binding capabilities (Fig. 2l). Collectively, $\alpha \mathrm{Fc}-\mathrm{NP}$ could serve as a versatile antibody immobilization platform by integrating different types of immunomodulatory mAbs conveniently and efficiently, without impairing their antigen-binding capability.

imNA $A_{\alpha P D 1} \&$ aPDL1 enhance $T$ cell-mediated cytotoxicity in vitro. We have successfully constructed a versatile antibody 
a

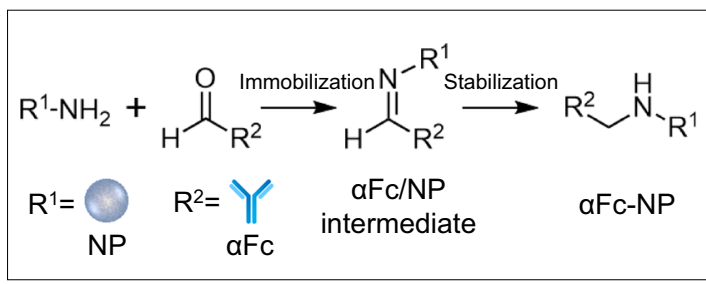

d
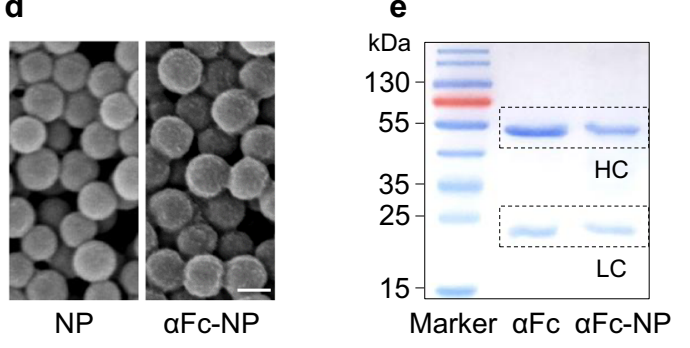

b

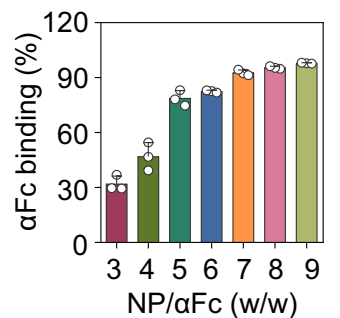

f

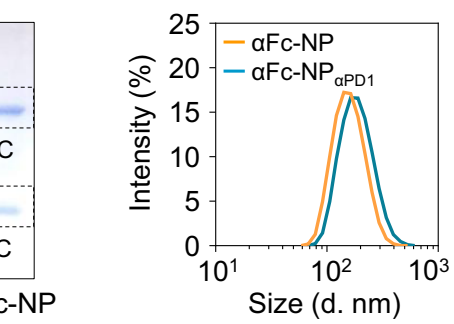

C

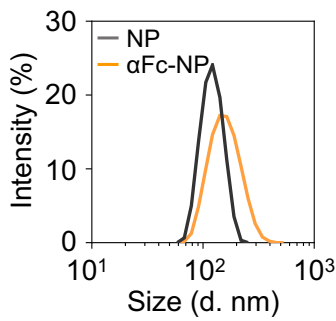

g

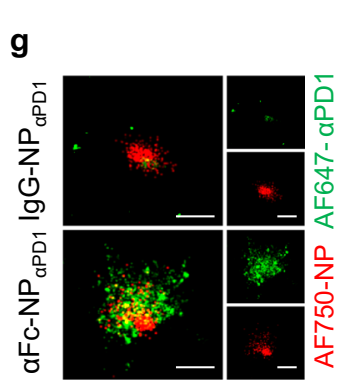

h
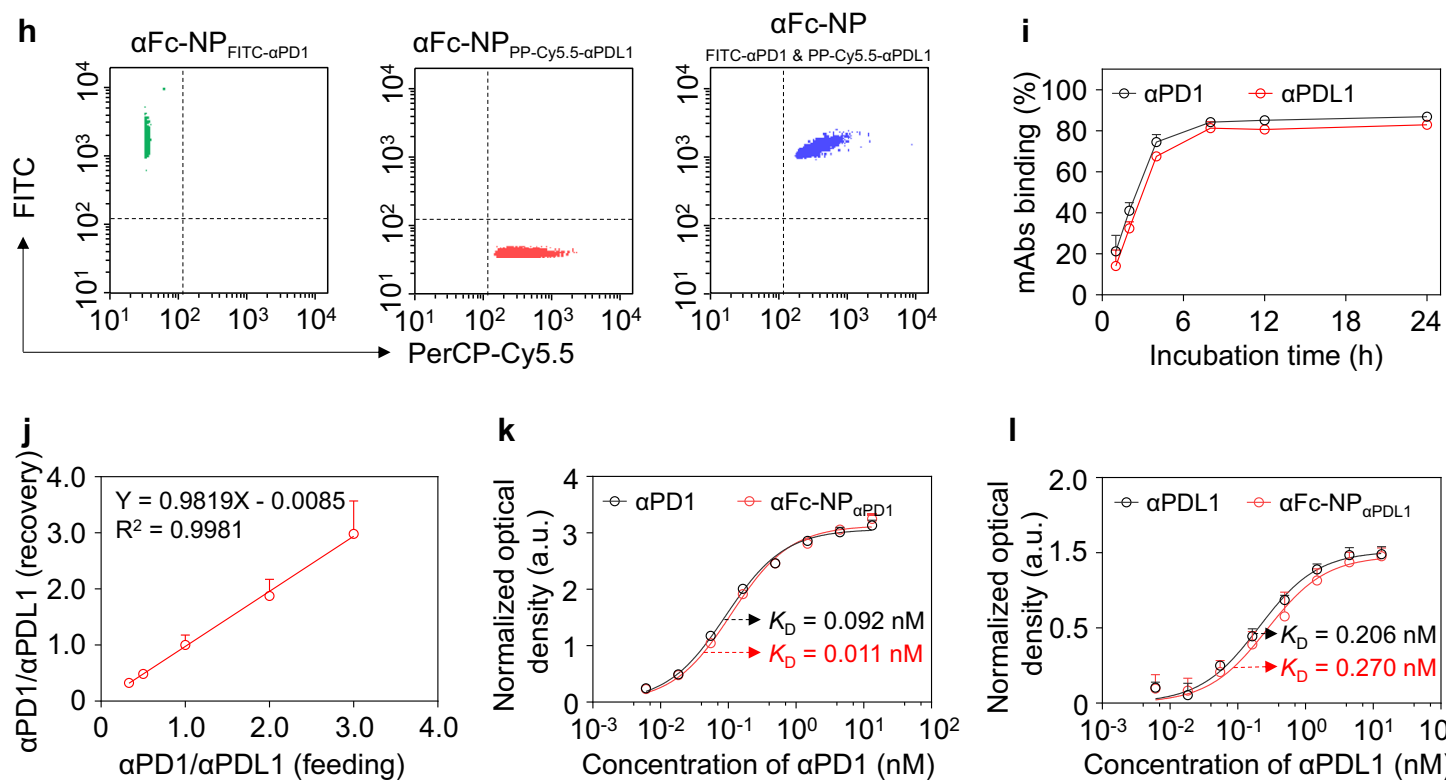

k

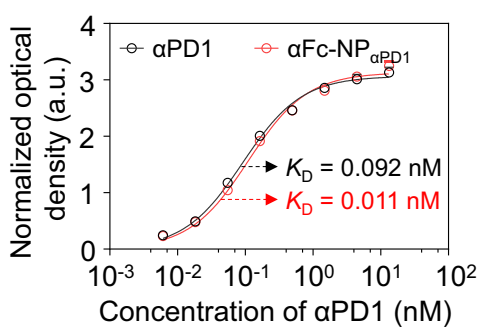

I

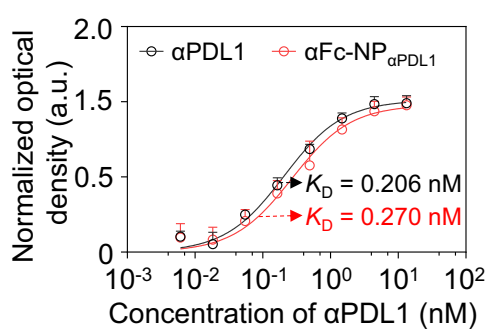

Fig. 2 Construction and characterization of the antibody immobilization platform ( $\alpha$ Fc-NP). a Schematic depicting the construction of $\alpha$ Fc-conjugated nanoparticles ( $\alpha \mathrm{Fc}-\mathrm{NP}$ ). $\alpha \mathrm{Fc}$ was oxidized and immobilized onto aminated polystyrene NP via aldol condensation. $\mathbf{b}$ Determination of the $\alpha \mathrm{Fc}$ binding efficacy by ELISA. c, Average hydrodynamic size of NP and $\alpha F c-N P$ as determined by DLS, confirming that NP had an average diameter of $123.4 \pm 4.1 \mathrm{~nm}$ and $\alpha F c-N P$ had an average diameter of $152.8 \pm 2.0 \mathrm{~nm}$. $\mathbf{d}$ Representative scanning electron microscopy (SEM) image of NP and $\alpha F c-N P$. Scale bar, $100 \mathrm{~nm}$. e Reducing SDS-PAGE gel stained with Coomassie Brilliant Blue showing the heavy chain (HC) and light chain (LC) released from soluble $\alpha F c$ or $\alpha F c-N P$. $\beta$ mercaptoethanol treatment breaks the interchain disulfide bonds and separates $\mathrm{HC}$ and LC of $\alpha \mathrm{FC}$. The molecular weights of $\mathrm{HC}$ and LC are approximately $50 \mathrm{kDa}$ and $25 \mathrm{kDa}$, respectively. $\mathbf{f}$ Size distribution of $\alpha \mathrm{Fc}-\mathrm{NP}$ and $\alpha \mathrm{Fc}-\mathrm{NP}_{\alpha \mathrm{PD} 1}$ determined by DLS, confirming the $\alpha \mathrm{Fc}_{\mathrm{N}} \mathrm{NP}_{\alpha \mathrm{PD} 1}$ had an average diameter of $176.1 \pm 5.3 \mathrm{~nm}, 30 \mathrm{~nm}$ larger than $\alpha F c-N P$. g Stochastic optical reconstruction microscopy (STORM) images of IgG-NP $\alpha$ PD1 and $\alpha F c-N P_{\alpha P D 1}$. NP and $\alpha P D 1$ were labeled with AF750 and AF647, respectively. An NP conjugating IgG control antibody (IgG-NP) was used as a control. Scale bar, $200 \mathrm{~nm}$. h Nanoflow Cytometry showed that $\alpha$ Fc-NP could simultaneously bind two types of mAbs. $\alpha$ Fc-NP was incubated with FITC-labeled $\alpha$ PD1 and PerCP-Cy5.5-labeled $\alpha P D L 1$ separately or in combination. i Binding efficacies of $\alpha$ PD1 and $\alpha$ PDL1 versus incubation time. $\alpha F c-N P$ were incubated with $\alpha P D 1$ and $\alpha P D 1$ at an $\alpha F c$ : $\alpha P D 1: \alpha P D L 1$ ratio of 1:0.5:0.5 for different periods, and then the unbound $\alpha P D 1$ and $\alpha$ PD1 were then examined by ELISA. $\mathbf{j}$ The ratio of $\alpha$ PD1 and $\alpha$ PDL1 immobilized by $\alpha F c-N P$. $\alpha F c-N P$ was incubated with $\alpha$ PD1 and $\alpha$ PDL1 at an $\alpha F c$ : ( $\alpha$ PD1 \& $\alpha$ PDL1) ratio of 1:1, while the ratio of $\alpha$ PD1 and $\alpha$ PDL1 ranged from 0.3 to 3.0. $\mathbf{k}$, I The capabilities of $\alpha \mathrm{Fc}-\mathrm{NP}_{\alpha \mathrm{PD} 1}$ and $\alpha \mathrm{Fc}-\mathrm{NP}_{\alpha \mathrm{PDL} 1}$ to bind corresponding antigens. Data are the means $\pm \mathrm{s}$.d of three different experiments with similar results. Source data are provided as a Source Data file.

immobilization platform, and we predicted that aFc-NP integrating two types of mAbs that targeted effector cells and tumor cells could serve as imNA, as they retained the basic function of the parental $\mathrm{mAbs}$ and could also engage the antigens on the surface of both cells and bridge them together like an 'adaptor'. To assess the superiority of imNA, $\mathrm{T}$ cell-mediated antitumor immunity was selected as an experimental model and four groups were established: (1) IgG isotype control (IgG control); (2) free $\alpha P D 1$ and $\alpha$ PDL1 (Free ${ }_{\alpha P D 1}$ \& $\alpha$ PDL1 $)$; (3) a physical mixture of $\alpha F c-N P$ integrating $\alpha \mathrm{PD} 1$ and $\alpha F c-N P$ integrating $\alpha \mathrm{PDL} 1$ $\left(\mathrm{NP}_{\alpha \mathrm{PD} 1} \& \mathrm{NP}_{\alpha \mathrm{PDL} 1}\right)$, and (4) aFc-NP simultaneously integrating aPD1 and $\alpha P D L 1\left(\right.$ imNA $\left._{\alpha \mathrm{PD} 1} \& \mathrm{aPDL}\right)$ (Fig. 3a). We first 


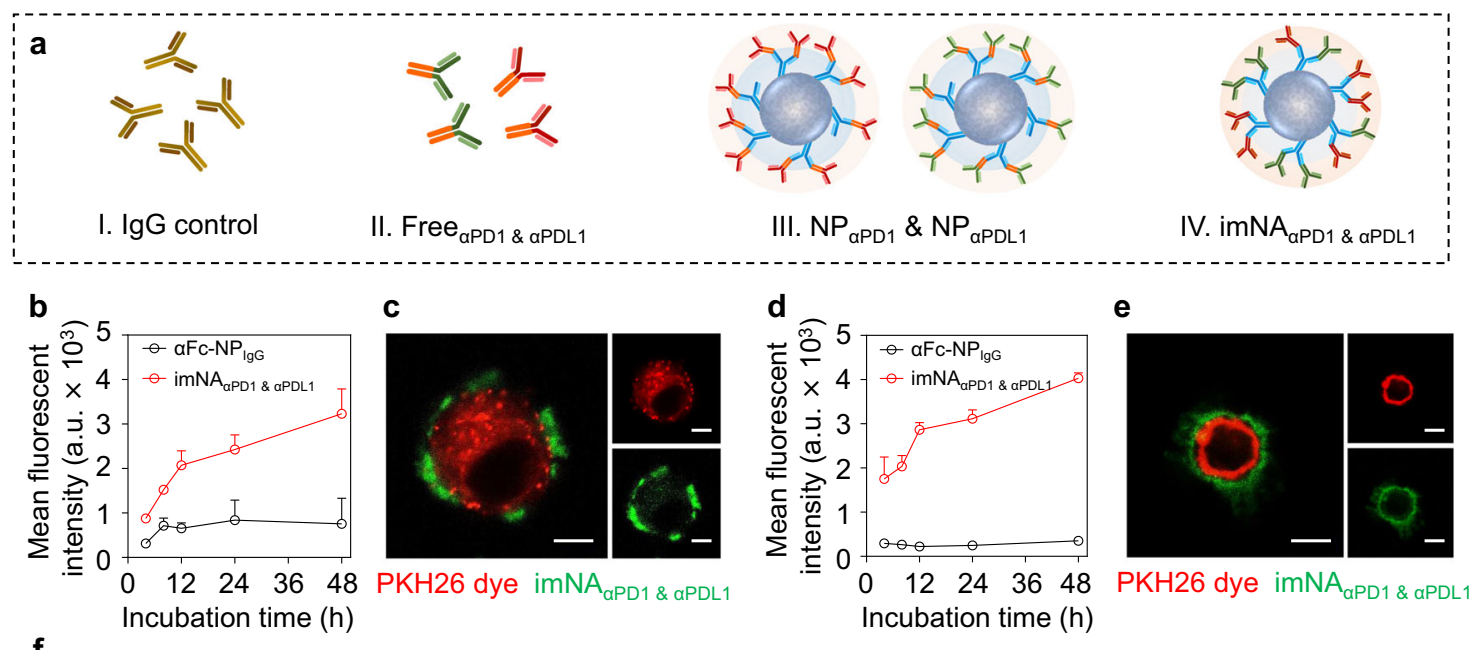

f

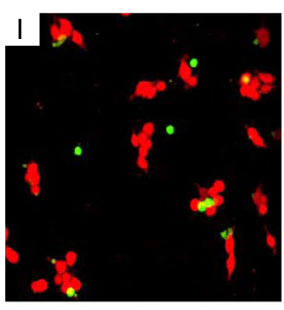

g
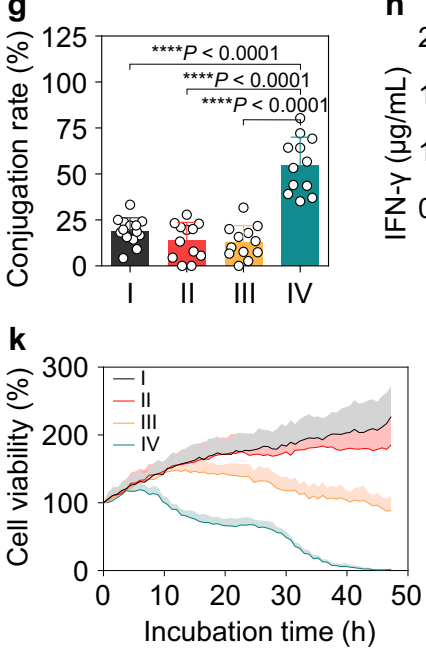

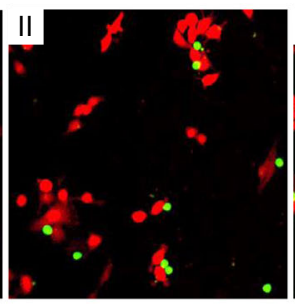

h

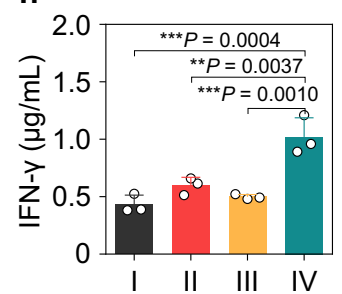

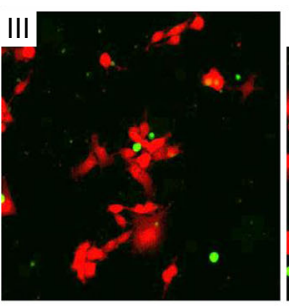

i

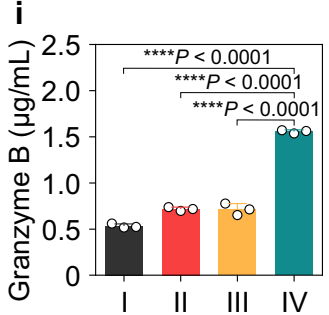

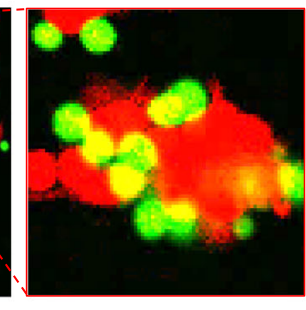

B16-F10 cell (mCherry)

j

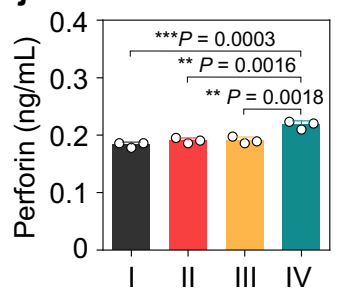

I
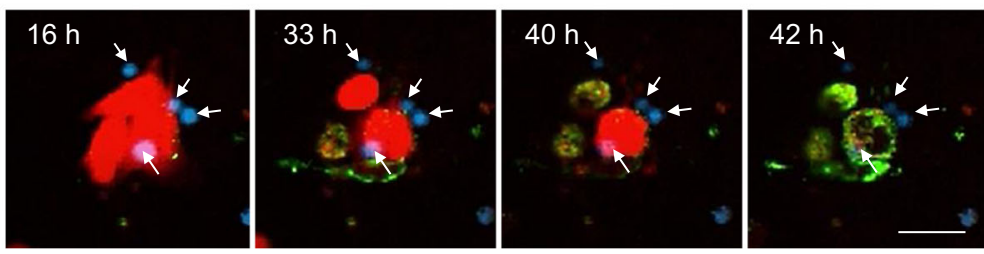

B16-F10 cell (mCherry) T cell (CellTrace Blue) Annexin V (FITC)

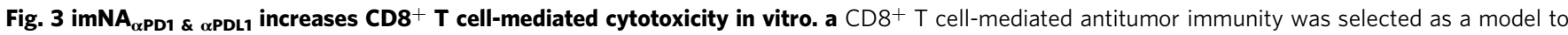

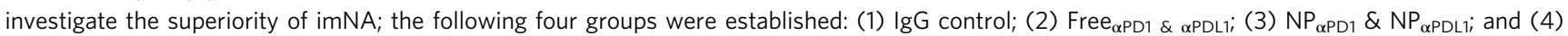
$i m N A_{\alpha P D 1} \& \alpha P D L 1 . b$ Extracellular fluorescence of B16-F10 cells after treatment with $\alpha F_{C}-N P_{\mid g G}$ or imNA $A_{\alpha P D 1} \& \alpha P D L 1$ for different periods. NPs were labeled with FITC. a.u., arbitrary unit. Data were presented as mean \pm s.d. $n=3$ biologically independent samples. c Confocal images of B16-F10 cells showed cell associations with imNA $\alpha$ PD1 \& $\alpha$ PDL1 1 after a $24 \mathrm{~h}$ incubation. The cell membrane was stained with PKH26 dye. Scale bar, $5 \mu \mathrm{m}$. $\mathbf{d}$ Extracellular fluorescence of $\mathrm{CD}^{+} \mathrm{T}$ cells after treatment with $\alpha \mathrm{Fc}-\mathrm{NP}_{\mathrm{IgG}}$ or imNA $\mathrm{APD} 1_{\alpha} \alpha \mathrm{PDL1}$ for different periods. Data were presented as mean $\pm \mathrm{s}$.d. $n=3$ biologically independent samples. e Confocal images of $\mathrm{CD}^{+} \mathrm{T}$ cells showing cell association with imNA $\mathrm{\alpha PD}_{1} \& \alpha \mathrm{PDL}$ after a $24 \mathrm{~h}$ incubation. Scale bar, $5 \mu \mathrm{m}$. $\mathbf{f}$ Confocal images showing the conjugations of $\mathrm{CD}^{+}{ }^{+} \mathrm{T}$ cells on B16-F10 tumor cells. CFSE-labeled CD8 ${ }^{+} \mathrm{T}$ cells and B16-F10-mCherry cells were co-incubated for $8 \mathrm{~h}$ in the presence of different treatments, and cell conjugations were detected after washing. Scale bar, $50 \mu \mathrm{m}$. $\mathbf{g}$ The conjugation rates were determined manually by observing the red/green fluorescence overlap in multiple nonoverlapping images. Data were presented as mean $\pm s . d$. $n=12$ nonoverlapping images. The concentrations of IFN- $\gamma(\mathbf{h})$, Granzyme B (i), and Perforin (j) in the supernatant of coincubated cells were examined by ELISA. CD8 ${ }^{+}$T cells and B16F10 cells (10:1) were coincubated and treated as indicated above for $24 \mathrm{~h}$. Data are presented as means \pm s.d. $n=3$ biologically independent samples.

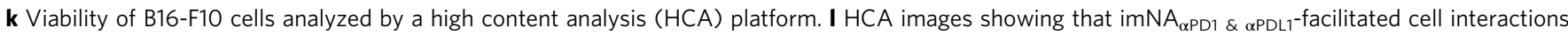
induced tumor cells apoptosis. Scale bar, $10 \mu \mathrm{m}$. Data are presented as means \pm s.d. Statistical significance was calculated via one-way ANOVA with the Tukey post-hoc test. ${ }^{\star} P<0.05 ;{ }^{\star \star} P<0.01 ;{ }^{\star \star \star} P<0.001 ;{ }^{\star \star \star \star} P<0.0001$. Source data are provided as a Source Data file. 
investigated the association of imNA $\mathrm{APD}_{\alpha} \& \alpha \mathrm{PDL} 1$ with tumor cells and CD8 ${ }^{+} \mathrm{T}$ cells. To mimic the tumor microenvironment, B16F10 melanoma cells and primary $\mathrm{CD}^{+} \mathrm{T}$ cells isolated from splenocytes were stimulated with interferon-gamma (IFN- $\gamma$ ) or anti-CD3/CD28 antibodies, and remarkable upregulation of PDL1 and PD1 was observed in B16-F10 cells and CD8 ${ }^{+} \mathrm{T}$ cells, respectively (Supplementary Fig. 7). Stimulated B16-F10 and $\mathrm{CD}^{+} \mathrm{T}$ cells were incubated with fluorescein isothiocyanate (FITC)-labeled imNA ${ }_{\alpha \mathrm{PD} 1} \&$ aPDL1 , and the targeting ability was assessed via flow cytometry and microscopy. As shown in Supplementary Fig. 8, the amount of imNA $_{\alpha \mathrm{PD} 1} \& \alpha \mathrm{PDL1}$ associated with B16-F10 cells increased with the extension of incubation time, as measured by the elevated median fluorescence intensity (MFI). Importantly, we confirmed that most imNA $\mathrm{A}_{\alpha \mathrm{PD} 1}$ \& aPDL1 was absorbed on the membrane but were not internalized into the cell via trypan blue quenching (Fig. $3 \mathrm{~b}$ and Supplementary Fig. 8). Confocal laser scanning microscopy (CLSM) images also revealed that a substantial amount of imNA $\mathrm{APD}_{1} \&$ aPDL1 adsorbed onto the cell surface (the membrane of B16-F10 cells was labeled with the red dye PKH26) (Fig. 3c) rather than becoming internalized.

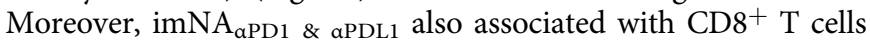
in a time-dependent manner (Fig. 3d and Supplementary Fig. 9), and few formulations were internalized into $\mathrm{CD}^{+} \mathrm{T}$ cells (Fig. 3e). In contrast, $\alpha \mathrm{Fc}-\mathrm{NP}$ integrating IgG control ( $\left.\alpha \mathrm{Fc}-\mathrm{NP}_{\mathrm{IgG}}\right)$ exhibited weak interaction with both tumor cells and CD8 ${ }^{+}$ $\mathrm{T}$ cells, indicating that the association of imNA $\mathrm{APD}_{\alpha} \&$ aPDL1 with cells was dependent on specific antibody-antigen recognition (Fig. 3b, d, and Supplementary Figs. 8 and 9), and these results confirmed that the well-known co-inhibitory molecules PD1 and PDL1 can also serve as binding sites for imNA $\alpha$ PD1 \& aPDL1. Additionally, FITC-labeled $\mathrm{NP}_{\alpha \mathrm{PDL} 1}$ and $\mathrm{NP}_{\alpha \mathrm{PD} 1}$ could only efficiently associate with B16-F10 cells and CD8 ${ }^{+} \mathrm{T}$ cells, but not both (Supplementary Fig. 10).

Having confirmed that imNA $\mathrm{APD}_{\mathrm{P} 1} \&$ aPDL1 can associate with tumor cells and $\mathrm{T}$ cells simultaneously, we further investigated whether imNA ${ }_{\alpha \mathrm{PD} 1} \&$ aPDL1 could promote the effector-target cell conjugation. mCherry-expressing B16-F10 cells (B16-F10mCherry) and carboxyfluorescein succinimidyl ester (CFSE)-labeled $\mathrm{CD}^{+} \mathrm{T}$ cells were coincubated in the presence of IgG control, Free $_{\alpha \mathrm{PD} 1 \& \alpha \mathrm{PDL} 1}, \mathrm{NP}_{\alpha \mathrm{PD} 1} \& \mathrm{NP}_{\alpha \mathrm{PDL} 1}$ or $\mathrm{imNA}_{\alpha \mathrm{PD} 1} \& \alpha \mathrm{PDL} 1$, and the conjugate formations were detected by confocal microscopy after washing suspended $\mathrm{CD}^{+} \mathrm{T}$ cells. As shown in Fig. $3 \mathrm{f}$, imNA $\mathrm{APD}_{\alpha}$ \& aPDL1 significantly increased the conjugation compared with other treatments, with approximately $60 \%$ of B16-F10 cells conjugated with one or more $\mathrm{CD}^{+} \mathrm{T}$ cells (Fig. 3g). As treatment with $\mathrm{NP}_{\mathrm{aPD} 1} \&$ $\mathrm{NP}_{\text {aPDL1 }}$ resulted in similar conjugate formations to IgG control and Free $_{\alpha \mathrm{PD} 1}$ \& $\alpha \mathrm{PDL1}$, we confirmed that imNA $\mathrm{APD1}_{\alpha}$ \& aPDL1 could simultaneously tightly engage tumor cells and CD8 ${ }^{+} \mathrm{T}$ cells, and physically link them together. These results highlighted the critical distinction between imNA $\mathrm{\alpha PD1}_{\text {\& } \alpha \mathrm{PDL} 1}$ and the mixture of soluble $\mathrm{mAbs}$ or NP integrated with monospecific mAbs.

To investigate whether imNA $\mathrm{APD}_{\mathrm{P} 1} \&$ aPDL1 could activate $\mathrm{T}$ cells in vitro, $\mathrm{CD}^{+} \mathrm{T}$ cells were coincubated with B16-F10 cells in a medium containing different forms of $\alpha \mathrm{PD} 1$ and $\alpha \mathrm{PDL} 1$, and cytokine production was examined at $24 \mathrm{~h}$ post-coincubation. imNA $_{\alpha \mathrm{PD} 1}$ \& $\alpha \mathrm{PDL1}^{-}$-treated cells showed the highest level of interferon-gamma (IFN- $\gamma$ ), which is a predictor of cytotoxic T lymphocyte (CTL)-mediated response ${ }^{31}$, compared with cells treated with equivalent amounts of Free ${ }_{\alpha \mathrm{PD} 1} \&$ aPDL1 or $\mathrm{NP}_{\alpha \mathrm{PD} 1} \&$ $\mathrm{NP}_{\alpha \mathrm{PDL} 1}$ (Fig. $3 \mathrm{~h}$ ). imNA $\mathrm{APD1}_{\alpha}$ aPDL1 also significantly increased the secretion of granzyme $\mathrm{B}$ and perforin ${ }^{32}$, the two main cytolytic granules released by CTLs (Fig. 3i, j). Next, a high content analysis (HCA) platform was utilized to determine the contribution of imNA $\mathrm{APD1}_{\text {\& }}$ aPDL1-facilitated cell interactions to $\mathrm{CD}^{+} \mathrm{T}$ cells cytotoxicity. Stimulated $\mathrm{CD} 8^{+} \mathrm{T}$ cells and B16-F10 cells were coincubated with the presence of different formulations and Annexin V-FITC, which is used to detect apoptotic cells. As revealed by HCA, Free $\alpha_{\alpha P 1} \& \alpha$ PDL1 and $\mathrm{NP}_{\alpha \mathrm{PD} 1} \& \mathrm{NP}_{\alpha \mathrm{PDL} 1}$ had minimal and slight effect on B16-F10 cell apoptosis (FITCpositive large cells), respectively, compared with IgG control. Intriguingly, imNA ${ }_{\alpha \mathrm{PD} 1} \& \mathrm{aPDL}$ treatment drastically induced tumor cell upon extended incubation time, and few living tumor cells existed $48 \mathrm{~h}$ post-incubation (Fig. 3k, Supplementary Movie 1-4, and Supplementary Fig. 11). HCA images confirmed that the enhanced T cell-mediated cytotoxicity could be attributed to the imNA $A_{\alpha \mathrm{PD} 1} \&$ aPDL1-facilitated interaction between $\mathrm{CD} 8^{+}$ $\mathrm{T}$ cells and tumor cells (Fig. 3l). Furthermore, we examined whether imNA $\alpha$ PD1 \& aPDL1 could enhance the cytotoxicity of antigen-specific $T$ cells by coculturing ovalbumin (OVA)-specific OT-1 CD8 ${ }^{+} \mathrm{T}$ cells and B16-F10-OVA cells in the presence of different formulations. As shown in Supplementary Fig. 12, imNA $_{\alpha \mathrm{PD} 1 ~ \& \text { aPDL1}}$-treated $\mathrm{CD} 8^{+} \mathrm{T}$ cells were more effective at killing B16-F10-OVA than those treated with a mixture of free antibodies or $\mathrm{NP}_{\alpha \mathrm{PD} 1} \& \mathrm{NP}_{\alpha \mathrm{PDL} 1}$. Collectively, these results confirmed that imNA $\mathrm{APD}_{\alpha} \&$ aPDL1 can promote the interaction between $\mathrm{CD}^{+} \mathrm{T}$ cells and tumor cells, and achieve an enhanced antitumor activity in vitro over the combination of monospecific mAbs.

imNA $A_{\alpha P D 1} \&$ aPDL1 enhances the antitumor effect of $T$ cells in vivo. Encouraged by the capability of imNA ${ }_{\alpha \mathrm{PD} 1} \& \mathrm{aPDL}_{1}$ to enhance $\mathrm{CD}^{+} \mathrm{T}$ cell-mediated cytotoxicity in vitro, we further assessed whether imNA $\mathrm{aPD1}_{\mathrm{P}} \mathrm{\alpha PDL1}$ could potentiate the antitumor effect of $\alpha$ PD1 and $\alpha$ PDL1 in vivo. Prior to the antitumor study, tumor enrichment of Free ${ }_{\alpha \mathrm{PD} 1 \& \alpha \mathrm{PDL} 1}$ and imNA $\mathrm{APD1}_{\alpha}$ aPDL1 was first investigated. BALB/c mice bearing $4 \mathrm{~T} 1$ breast tumors were intravenously administered with soluble or NP-immobilized Cy5labeled aPD1 \& aPDL1; tumor tissues were collected at predetermined time points, and the $\mathrm{mAb}$ signals were monitored using a fluorescence in vivo imaging system (IVIS). As shown in

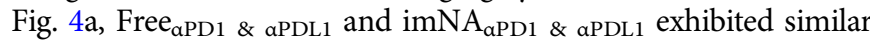
tumor accumulation at $12 \mathrm{~h}$ and $24 \mathrm{~h}$ post-injection. Notably, unlike the quick clearance of free mAbs, imNA $\mathrm{APD}_{\alpha} \&$ aPDL1 continued to accumulate at the tumor sites after $24 \mathrm{~h}$ and were retained after more than $72 \mathrm{~h}$. The imNA $\mathrm{APD}_{\mathrm{a} 1}$ \& aPDL1 group showed $\sim 100.3 \%$ and $\sim 936.9 \%$ higher fluorescence intensity than the Free ${ }_{\alpha \mathrm{PD} 1} \& \alpha \mathrm{\alpha DL} 1$ group at $48 \mathrm{~h}$ and $72 \mathrm{~h}$, respectively (Fig. $4 \mathrm{~b}$ ). The intratumoral distribution of mAbs was also examined using immunofluorescence staining, and much stronger fluorescent signals were detected in the imNA $_{\alpha \mathrm{PD} 1} \&$ aPDL1 group than the Free $_{\alpha \mathrm{PD} 1} \& \alpha \mathrm{\alpha PL} 1$ group at $48 \mathrm{~h}$ and $72 \mathrm{~h}$, consistent with the results of IVIS imaging (Supplementary Fig. 13). Furthermore, mice bearing GFP-expressing 4T1 murine breast tumors were treated with Free $\alpha_{\alpha P D 1} \& \alpha$ PDL1 or imNA ${ }_{\alpha P D 1} \& \alpha$ PDL1, and tumor tissues were harvested, sectioned, and stained with anti-CD8 antibody for confocal laser scanning microscopy (CLSM). As shown in Fig. 4c, $\mathrm{CD}^{+} \mathrm{T}$ cells (red) were mainly located in the space between tumor cells (green) in the Free ${ }_{\alpha \mathrm{PD} 1} \& \alpha \mathrm{PDL}_{1}$-treated tumors, while most $\mathrm{CD}^{+} \mathrm{T}$ cells were closely associated with tumor cells in the imNA $_{\alpha \mathrm{PD} 1}$ \& aPDL1-treated group (as indicated by the white arrows), which was accompanied by enhanced cytotoxicity against tumor cells (measured by the destruction of cell integrity). These results confirmed that $\mathrm{imNA}_{\alpha \mathrm{PD} 1} \&$ aPDL1 can efficiently promote the engagement between $\mathrm{T}$ cells and tumor cells in vivo.

Next, an aggressive, hard-to-treat B16-F10 melanoma tumor model was established, and tumor-bearing mice were treated with IgG control, Free FPD1 \& $\alpha$ PDL1 $_{1} \mathrm{NP}_{\alpha \mathrm{PD} 1} \& \mathrm{NP}_{\alpha \mathrm{PDL} 1}$ or imNA ${ }_{\alpha \mathrm{PD} 1} \&$ aPDL1 following a $\mathrm{q} 3 \mathrm{dx} 3$ course (three times at intervals of three days) (Fig. 4d). As shown in Fig. 4e, f, Free $_{\alpha \mathrm{PD} 1} \& \alpha \mathrm{PDL} 1$, and $\mathrm{NP}_{\alpha \mathrm{PD} 1} \& \mathrm{NP}_{\alpha \mathrm{PDL} 1}$ only slightly suppressed tumor growth, with $37.8 \pm 22.8 \%$ and $50.7 \pm 25.50 \%$ 
a

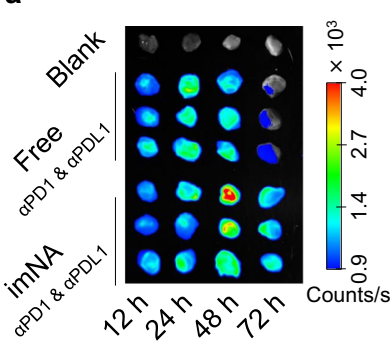

b

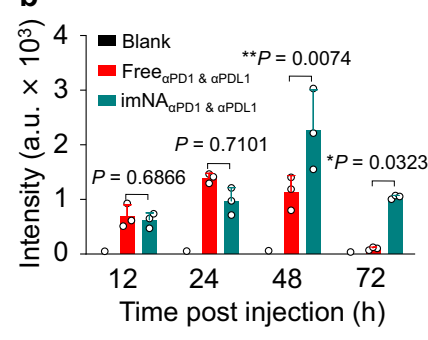

c

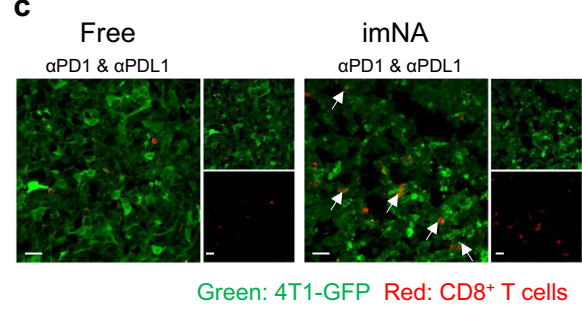

d

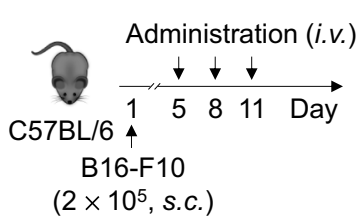

g

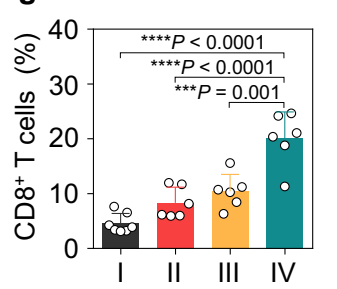

k

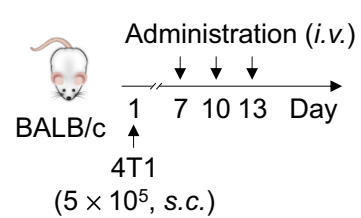

n

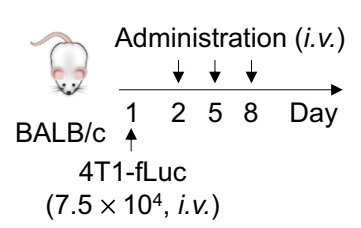

e

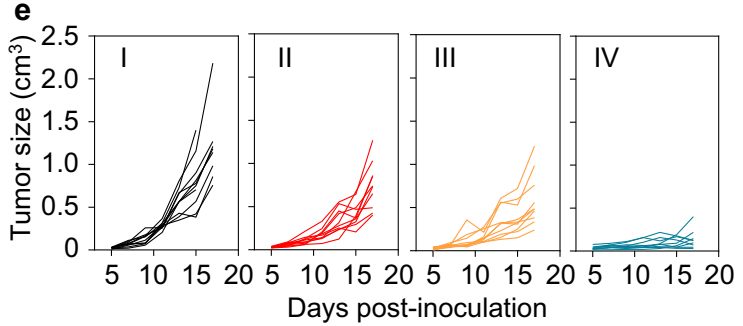

$\mathbf{h}$
$\frac{0}{0}$
0
$\overline{0}$
0
$\vdash$
$+\infty$
0
0
$+\infty$
$\frac{1}{\pi}$
$\frac{0}{0}$
$\mathbf{1}$

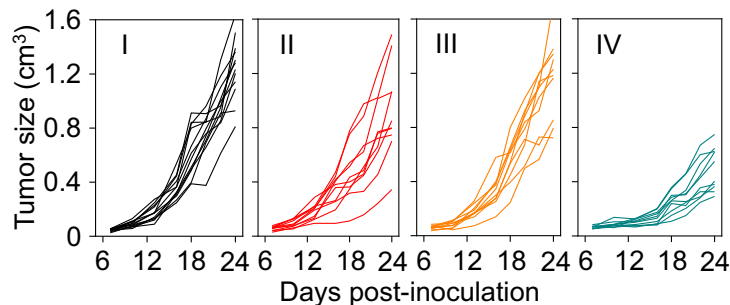

0

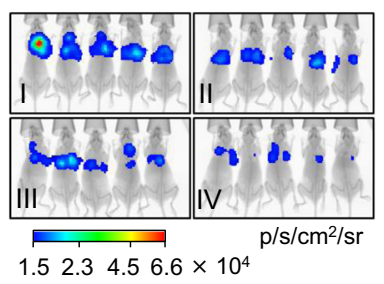

I. IgG control i

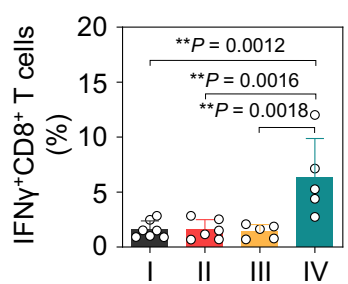

p

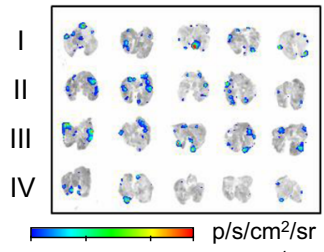

$0.10 .470 .831 .2 \times 10^{4}$

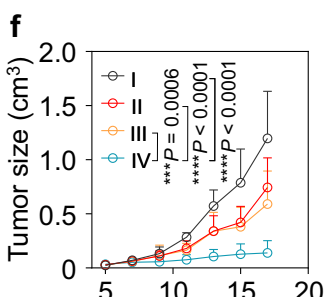

Days post-inoculation
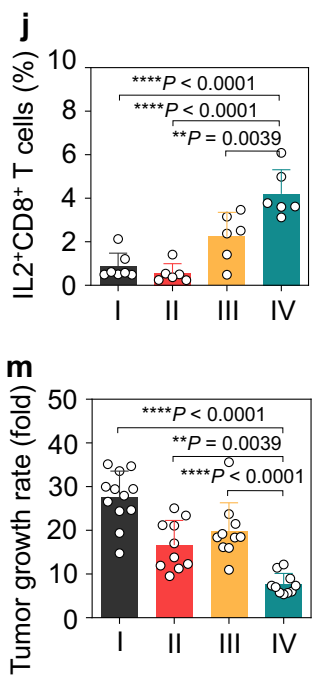

q

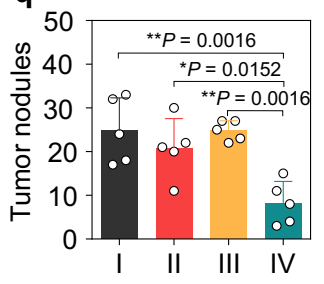

IV. imNA $A_{\alpha P D 1} \& \alpha P D L 1$

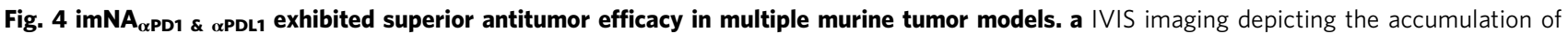
imNA ${ }_{\alpha P D 1} \& \alpha P D L 1$ in $4 T 1$ tumors. $\mathbf{b}$ Quantification of mAb accumulation in tumor tissues. Data are presented as means \pm s.d. $n=3$ biologically independent mice. Statistical significance was calculated via two-way ANOVA followed by the Tukey post-hoc test. ${ }^{\star} P<0.05$; ${ }^{\star \star} P<0.01$. $\mathbf{c}$ Engagement of 4T1-GFP tumor cells and CD8 ${ }^{+}$T cells in tumor tissues. Scale bars, $200 \mathrm{~mm}$. d Experimental protocol for the B16-F10 melanoma model used in e-g, the equivalent injection dose of $\alpha$ PD1 and $\alpha$ PDL1 was $2.5 \mathrm{mg} / \mathrm{kg}$. Individual (e), and average (f) tumor growth curves of B16-F10 tumors in different groups. Data are presented as means \pm s.d. $n=10$ biologically independent mice. $\mathbf{g}$ The abundance of $\mathrm{CD} 8^{+} \mathrm{T}$ cells in tumor tissues at the end of treatment. Data are presented as means \pm s.d. $n=6-7$ biologically independent mice (IgG control: $n=7$; other groups: $n=6$ ). Flow cytometry to evaluate the percentages of granzyme-secreting (h), IFN- $\boldsymbol{\gamma}$-secreting (i), and IL-2-secreting CTLs (j) in melanoma tumors. Data are presented as means \pm s.d. $n=5-7$ biologically independent mice. For $\mathbf{h}$, imNA $\mathrm{APD} 1_{\alpha} \alpha \mathrm{PDL} 1: n=5$, other groups: $n=6$; for $\mathbf{i}, \operatorname{lgG}$ control: $n=7$, Free ${ }_{\alpha \mathrm{PD} 1} \& \alpha \mathrm{PDL} 1: n=6$, other groups, $n=5$; for $\mathbf{j}$, IgG control: $n=7$, other groups, $n=6$. $\mathbf{k}$ Experimental protocol for the $4 \mathrm{~T} 1$ breast cancer model used in $\mathbf{I}$-m. I Individual tumor growth curves. $\mathbf{m}$ Tumor growth rates are shown in (I) at 24 days post-inoculation. Data are presented as means \pm s.d. $n=10-12$ biologically independent mice ( $\operatorname{lgG}$ control: $n=12$; other groups: $n=10$ ). $\mathbf{n}$ Experimental protocol for the lung metastatic breast cancer model used in o-q. $\mathbf{0}$ In vivo bioluminescence imaging (BLI) of pulmonary metastases in mice at 16 days post-injection. $\mathbf{p}$ Ex vivo BLI of pulmonary metastases. $\mathbf{q}$ Tumor nodules on the lung tissues. Data are presented as mean \pm s.d. $n=5$ biologically independent mice. Statistical significance was calculated via one-way ANOVA with the Tukey post-hoc test. ${ }^{\star} P<0.05 ;{ }^{\star \star} P<0.01 ;{ }^{\star \star \star} P<0.001 ;{ }^{\star \star \star \star} P<0.0001$. Source data are provided as a Source Data file. 
inhibition rates versus the IgG control group at 18 days postinoculation, respectively. In marked contrast, tumor growth in the $\mathrm{imNA}_{\alpha \mathrm{PD} 1}$ \& aPDL1 treated group was dramatically delayed and led to 4.3-fold and 3.2-fold smaller tumors compared with

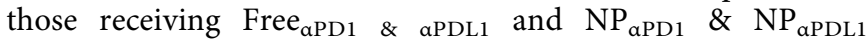
treatments, respectively. It is noteworthy that the physical mixture of $\mathrm{NP}_{\alpha \mathrm{PD} 1}$ and $\mathrm{NP}_{\alpha \mathrm{PDL} 1}$ exhibited limited antitumor efficacy compared with imNA ${ }_{\alpha \mathrm{PD} 1} \&$ aPDL1 , further corroborating the importance and necessity of immobilizing two mAbs onto a single NP. In addition, compared with the IgG control group, all the treatments improved median survival time of tumor-bearing mice, leading to a significantly longer time to endpoint in imNA $\mathrm{APD1}_{\alpha}$ aPDL1 group (Supplementary Fig. 14). Additionally, the selected $\mathrm{mAb}$ doses were well-tolerated in animal safety studies without noticeable weight loss during the treatment course (Supplementary Fig. 15).

To elucidate the mechanism by which imNA $\alpha$ PD1 \& $\alpha$ PDL1 achieved improved antitumor activity, we sought to examine the frequency of the $\mathrm{T}$ cell subpopulation in tumor tissues. As shown in Fig. $4 \mathrm{~g}$ and Supplementary Fig. 16, the frequency of CTLs $\left(\mathrm{CD} 45^{+} \mathrm{CD} 3{ }^{+} \mathrm{CD}^{+} \mathrm{T}\right.$ cells) in imNA ${ }_{\alpha \mathrm{PD} 1} \&$ aPDL1-treated tumors was 4.7-, 2.31-, and 1.81-fold higher than that of the IgG control, Free $\alpha_{\alpha \mathrm{PD} 1} \& \alpha_{\mathrm{PDL} 1}$, and $\mathrm{NP}_{\alpha \mathrm{PD} 1} \& \mathrm{NP}_{\alpha \mathrm{PDL} 1}$ groups, respectively. Meanwhile, imNA ${ }_{\alpha \mathrm{PD} 1} \&$ aPDL1 dramatically reduced the percentage of regulatory $\mathrm{T}$ cells (Tregs) (Supplementary Fig. 17), and the elevated $\mathrm{CD}^{+} \mathrm{T}$ cell/Treg ratio indicated that the $\mathrm{imNA}_{\alpha \mathrm{PD} 1} \&$ aPDL1 treatment could reverse the immunosuppressive microenvironment (Supplementary Fig. 18). More importantly, ex vivo phorbol 12-myristate 13-acetate/ ionomycin (PMA) restimulation of $\mathrm{T}$ cells revealed that imNA $_{\alpha \mathrm{PD} 1} \&$ aPDL1 could induce a substantial increase of Granzyme B-, IFN- $\gamma$ (interferon-gamma)- and IL-2 (interleukin-2)-secreting $\mathrm{CD}^{+} \mathrm{T}$ cells relative to the other treatments, suggesting the enhanced antitumor functionality and proliferation of CTLs in imNA ${ }_{\alpha \mathrm{PD} 1} \&$ aPDL1-treated tumors (Supplementary Fig. 19 and Fig. $4 \mathrm{~h}-\mathrm{j}$ ). We also found that $\mathrm{T}$ cells played a

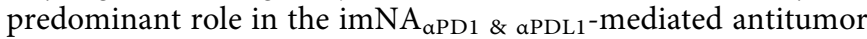
effect, while other PD1-expressing cells, including NK cells and DCs, played negligible roles (Supplementary Figs. 20 and 21). Furthermore, the PDL1-deficient B16-F10 cell line (PDL1-KOB16-F10 cells) was constructed using CRISPR-Cas9 technology (Supplementary Fig. 22a, b). Notably, both Free ${ }_{\alpha P D} \& \alpha$ PDL1 and imNA $_{\alpha \mathrm{PD} 1 \& \alpha \mathrm{PDL} 1}$ exhibited marginal benefits in terms of tumor control in the subcutaneous PDL1-KO-B16-F10 model (Supplementary Fig. 22c), confirming the importance of PDL1 on tumor cells in the imNA-mediated anti-tumor response and the importance of imNA ${ }_{\alpha \mathrm{PD} 1}$ \& $\alpha \mathrm{PDL} 1-$ facilitated cell interaction in tumor therapy.

With the confirmation of the anti-melanoma effect, we further explored the general applicability of imNA $_{\alpha \mathrm{PD} 1 \& \text { aPDL1 }}$ using a murine 4T1 mammary tumor model, which emulates stage IV human breast cancer and is otherwise unresponsive to anti-PD1/ PDL1 treatment ${ }^{33}$. Mice bearing orthotopic 4T1 tumors were treated as indicated above when the tumor volumes reached approximately $50 \mathrm{~mm}^{3}$ (Fig. 4k). At an equivalent injection dose, Free $_{\alpha \mathrm{PD} 1 \text { \& } \alpha \mathrm{PDL} 1}, \mathrm{NP}_{\alpha \mathrm{PD} 1} \& \mathrm{NP}_{\alpha \mathrm{PDL} 1}$ exhibited marginal benefits in terms of tumor control (Fig. 4l). Encouragingly, imNA $\mathrm{aPD1}_{\text {\& } \alpha \mathrm{PDL}^{-}}$ treated mice showed an enhanced response rate (Fig. 4l) and a reduced tumor growth rate (Fig. $4 \mathrm{~m}$ ) compared to the control treatment; meanwhile, the changes in body weight during treatment were within acceptable limits (Supplementary Fig. 23). Furthermore, the potential of imNA ${ }_{\alpha \mathrm{PD} 1} \& \alpha \mathrm{PDL1}$ to eliminate circulating tumor cells and inhibit tumor metastasis was evaluated in a pulmonary metastatic model. Mice were intravenously injected with $4 \mathrm{~T} 1$ cells expressing firefly luciferase (4T1fLuc) and then received a $\mathrm{q} 3 \mathrm{dx} 3$ course from the second day
(Fig. 4n). From the in vivo and ex vivo bioluminescence imaging results, in mice treated with PBS, Free $\alpha_{\mathrm{PDD} 1} \&$ aPDL1 and $\mathrm{NP}_{\alpha \mathrm{PD} 1} \&$ $\mathrm{NP}_{\alpha \mathrm{PDL} 1}$, bioluminescence signals were evident on 16 days postinfusion, in contrast, mice treated with imNA ${ }_{\alpha \mathrm{PD} 1} \&$ aPDL1 exhibited the weakest bioluminescence signals (Fig. 4o, p). Direct observation of whole lungs and hematoxylin-eosin (H\&E) staining verified the significant decrease in the number and size of metastatic nodules in the imNA ${ }_{\alpha \mathrm{PD} 1} \&$ aPDL1-treated group (Fig. 4q, and Supplementary Figs. 24, 25). The superior antimetastatic effects of imNA $\alpha$ PD1 \& $\alpha$ PDL1 can be partly

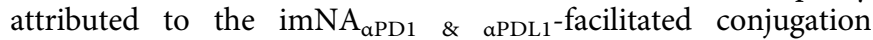
of $\mathrm{CD}^{+} \mathrm{T}$ cells and tumor cells in lung tissues and peripheral blood. Collectively, the enhanced antitumor efficacy of imNA ${ }_{\alpha \mathrm{PD} 1} \&$ aPDL1 in multiple tumor models indicated that imNA could extend the therapeutic potential of PD1/PDL1 blockade to a broader range of tumor types.

After confirming the remarkable antitumor effect of imNA $\mathrm{APD}_{\alpha}$ \& $\alpha \mathrm{PDL} 1$ in a variety of tumor models, we further investigated its biological safety. Male C57BL/6 mice (6-8 weeks

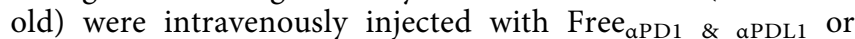

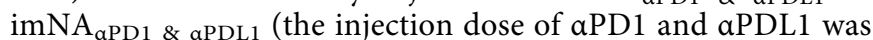
$2.5 \mathrm{mg} / \mathrm{kg}$ ) every three days, with three replicates. Six weeks following the final injection, mice were sacrificed to collect peripheral blood and the main organs. Multiple indices of liver function and renal function were examined. As shown in Supplementary Fig. 26, imNA $\mathrm{aPD1}_{\alpha}$ \& aPDL1 treatment did not significantly impair liver or renal function. The main organs (including liver, spleen, lung, kidney colon, and intestine) were subjected to $H \& E$ staining and minor to-no lesions in these organs were observed via histological analyses (Supplementary Fig. 27).

imNAs potentiate the antitumor effect of multiple immunomodulatory mAbs. Having shown that imNA $\mathrm{APD}_{\text {\& }}$ aPDL1 could significantly enhance the therapeutic efficacy of $\alpha$ PD1 and $\alpha$ PDL1 in vitro and in vivo, we next explored the possibility that imNAs could improve the antitumor efficiency of mAbs targeting immunomodulatory molecules expressed by tumor cells and other immune cells (for example, NK cells and macrophages). NK cells are potent cytotoxic lymphocytes of the innate immune system, and their activity is regulated by a repertoire surface receptor that recognizes their respective ligands on target cells ${ }^{34,35}$. Here, an NK cell-targeting imNA was constructed by gently mixing $a \mathrm{Fc}-\mathrm{NP}$ with anti-KLRG1 (killer-cell lectin-like receptor G1) antibody (aKLRG1) and anti-PDL1 antibody ( $\alpha$ PDL1); the former could specifically blockade cadherin/KLRG1 interaction and enhance the cytolytic activity and proliferation of NK cells, and the latter can bind to tumor cells. To evaluate the superiority of imNA ${ }_{\mathrm{aKLRG1}}$ \& aPD1 , a pulmonary metastatic model was established by i.v. injecting B16-F10 cells into C57BL/6 mice.

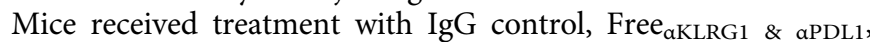

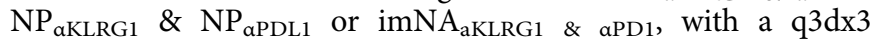
course, and the administration doses of aKLRG1 and aPD1 were $1.5 \mathrm{mg} / \mathrm{kg}$ (Fig. 5a, b). Lungs from treated mice were harvested at 20 days post-injection. The observation of whole lungs (Fig. $5 \mathrm{c}$ ) and calculation of metastatic nodules (Fig. 5d) showed that the formation of metastatic foci was effectively inhibited in the

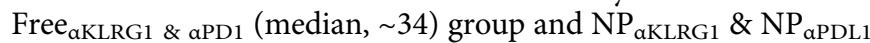
(median, $\sim 27$ ) group, compared with the control group (median, $\sim 62$ ). Encouragingly, very few metastatic foci (median, 7 ) were observed in the lung tissues of imNA ${ }_{\alpha K L R G 1 ~ \& ~ a P D L 1}$-treated mice. $\mathrm{H} \& \mathrm{E}$ staining confirmed the significant decrease in the number and size of metastatic nodules in the imNA ${ }_{\alpha K L R G 1 ~ \& ~ a P D L 1}$ group (Fig. 5e). We further confirmed that the NK cells play a predominant role in the imNA $\mathrm{aKLRG1}_{\text {\& }}$ aPDL1-mediated antitumor 


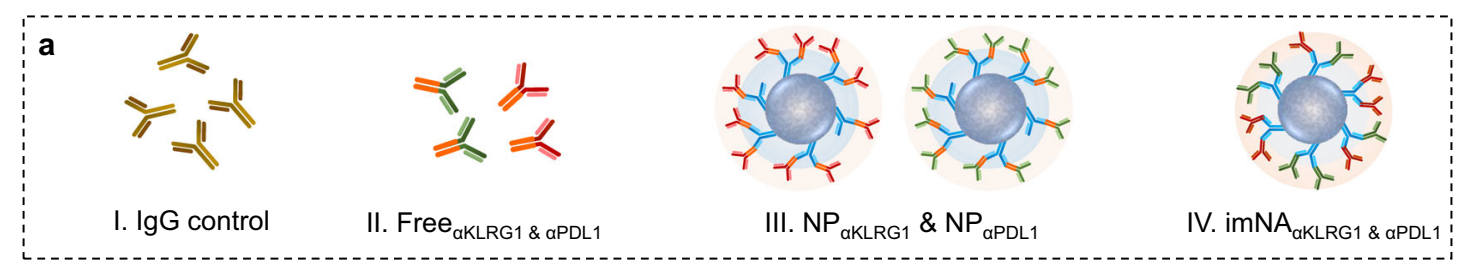

b

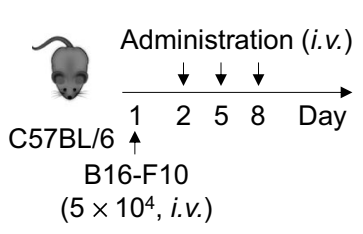

C

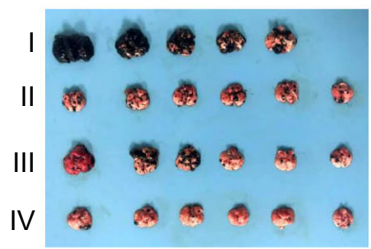

d

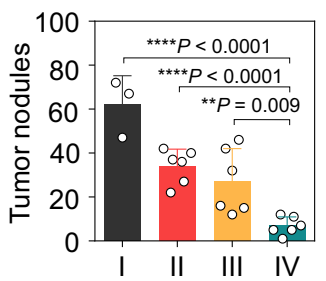

e

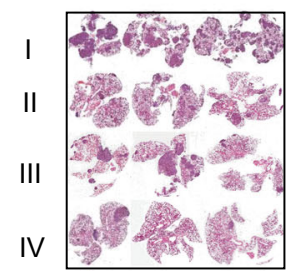

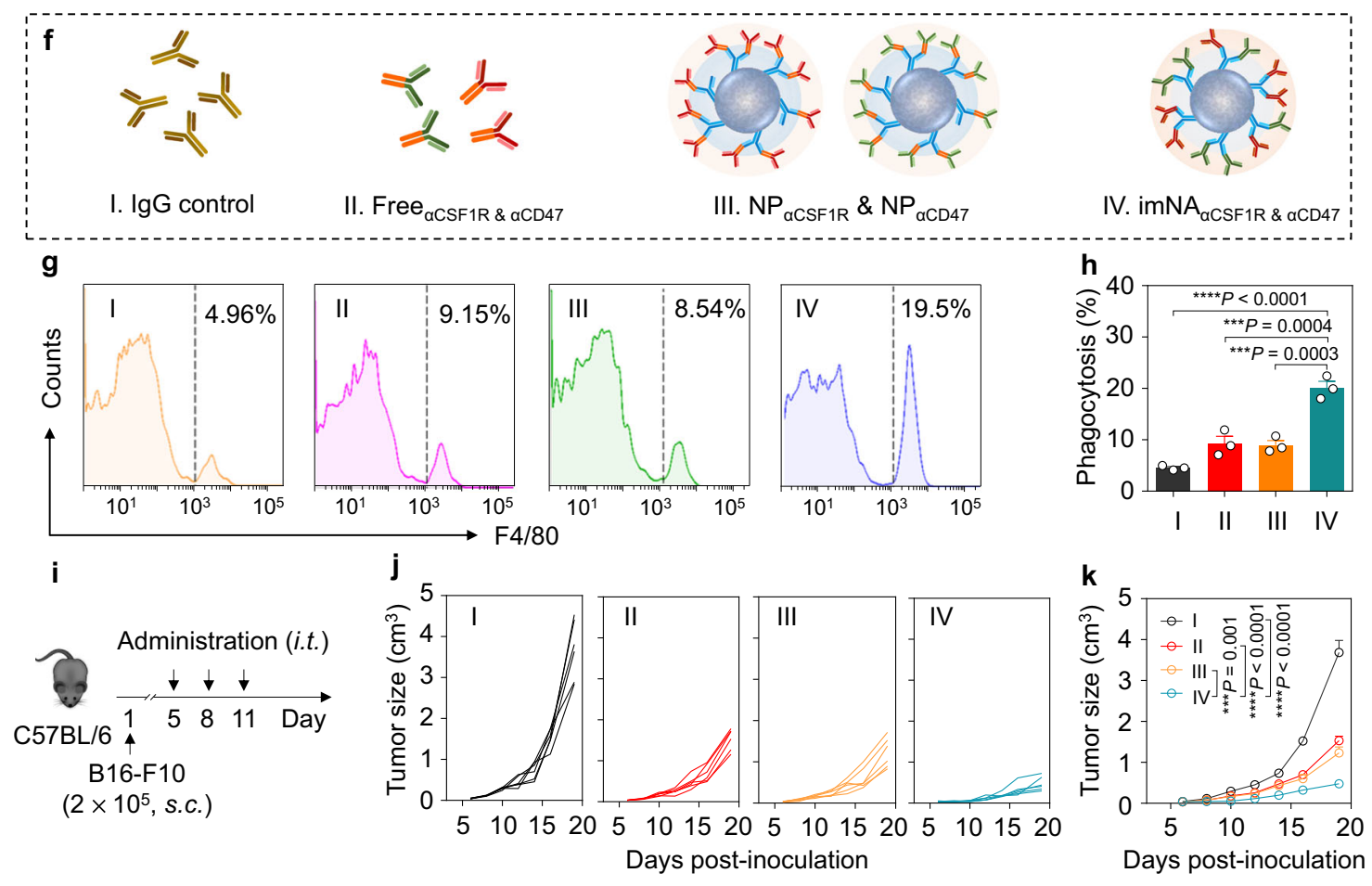

Fig. $5 \alpha F c-N P$ serves as a versatile antibody immobilization platform and imNAs enhance the anti-tumor effect of multiple immunomodulatory mAbs. a imNA was constructed to enhance the antitumor activity of NK cells, and the following four groups were established: (1) IgG control; (2) Free ${ }_{\alpha K L R G 1} \& \alpha P D L 1 ;$ (3) $\mathrm{NP}_{\alpha K L R G 1} \& \mathrm{NP}_{\alpha \mathrm{PDL} 1}$; and (4) imNA $\mathrm{AKLRG1}_{\alpha \alpha P D L 1}$. b Experimental protocol for the lung metastatic melanoma tumor model used in c-e: C57BL/6 mice were injected with $5.0 \times 10^{4}$ B16-F10 melanoma cells via the tail vein, and treatment started on the second day. The equivalent dose of $\alpha$ KLRG1 and $\alpha$ PDL1 was 1.5 $\mathrm{mg} / \mathrm{kg}$. c Images of lung tissues at 20 days post tumor cell infusion. $\mathbf{d}$ Calculation of tumor nodules on the lung tissues. Data are presented as means $\pm \mathrm{s}$.d. $n=3$ or 6 biologically independent mice, each pot represents one mouse. e Representative H\&E images of lung tissues. $\mathbf{f}$ imNA was constructed to enhance

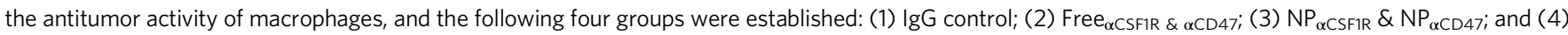
$\operatorname{imNA}_{\alpha C S F 1 R} \& \alpha C D 47$. Representative flow cytometric analysis images $(\mathbf{g})$ and relative quantification (h) of the phagocytosis of cancer cells by bone marrowderived macrophages (BMDMs). CFSE-labeled B16-F10 cells were incubated with BMDMs for $4 \mathrm{~h}$ in the presence of IgG control, Free $\alpha$ CSF1R \& $\alpha$ CD47, NP ${ }_{\alpha C S F 1 R} \&$ $\mathrm{NP}_{\alpha \mathrm{CD} 47}$ or imNA $\mathrm{\alpha CSF1R}_{\alpha} \alpha \mathrm{CD} 47$, and then subjected to flow cytometric detection. Phagocytosis was quantified as the percentage of CFSE-positive BMDMs. Data are presented as means \pm s.d. $n=3$ biologically independent samples. $\mathbf{i}$ Experimental protocol for the B16-F10 melanoma model used in $\mathbf{j}$, $\mathbf{k}$, the equivalent dose of $\alpha$ CSF1R and $\alpha C D 47$ was $1.5 \mathrm{mg} / \mathrm{kg}$, and the dose of $\mathrm{lgG}$ control was $3.0 \mathrm{mg} / \mathrm{kg}$. Individual (j) and average (k) tumor growth curves of B16F10 tumors in mice receiving different treatments. Data are presented as means \pm s.d. $n=6$ biologically independent mice. Statistical significance was calculated via one-way ANOVA with the Tukey post-hoc test. ${ }^{\star \star} P<0.01 ;{ }^{\star \star \star} P<0.001$; ${ }^{\star \star \star \star} P<0.0001$. Source data are provided as a Source Data file.

response using the NK cell depletion experiments (Supplementary Fig. 28).

Tumor-associated macrophages (TAMs) have attracted substantial attention in recent years, as they play a key role in tumor metastasis and therapeutic resistance ${ }^{7}$. TAMs are considerably plastic and can be either tumor-supportive (M2-like cells) or tumoricidal (M1-like cells). M2-like cells prevail in nearly all tumor types but have a limited ability to phagocytose tumor cells $^{36}$. Additionally, cancer cells always express a 'don't eat me' molecule, CD47, that binds to the signal regulatory protein a (SIRPa) receptor on macrophages and inhibits phagocytic activity $^{37}$. We proposed that skewing the M2-like phenotype 
towards an M1-like lineage, thus blocking the 'don't eat me' signal, and physically linking macrophages and tumor cells together could improve tumor cell engulfment. Thus, we immobilized aCSF1R (stimulating factor 1-receptor), which can inhibit CSF1/CSF1R signaling and repolarize the macrophage phenotype $^{38}$, and aCD47 (anti-CD47 antibody) onto $\alpha \mathrm{Fc}-\mathrm{NP}$, to form a macrophage-targeting imNA (imNA ${ }_{\alpha \operatorname{CSF} 1 \mathrm{R}} \& \mathrm{aCD}_{4}$ ) (Fig. 5f). To evaluate the advantage of imNAaCSF1R \& aCD47, we first cocultured CFSE-labeled B16-F10 cells and BMDMs (bone marrow-derived macrophages) in the presence of different therapeutics (Fig. 5g) and examined phagocytosis using flow cytometry. As shown in Fig. 5h, treatment with aCSF1R \& aCD47 and $\mathrm{NP}_{\alpha \mathrm{CSF} 1 \mathrm{R}} \& \mathrm{NP}_{\alpha \mathrm{CD} 47}$ could improve phagocytosis as measured by the CFSE signal in the macrophages. Of note, the

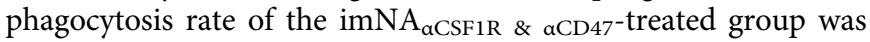
substantially higher than those of the other groups. More importantly, imNA $\mathrm{aCSF} 1 \mathrm{R}_{\mathrm{C}}$ \& $\mathrm{\alpha CD}_{7}$-facilitated phagocytosis of tumor cells by macrophages can efficiently increase the cognate APC (antigen-presenting cell) function of macrophages and stimulate the clonal expansion of tumor-specific T cells (Supplementary Fig. 29).

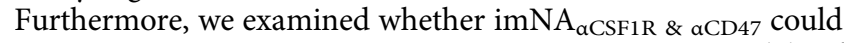
promote anti-tumor activity in vivo. Syngeneic mouse models of B16-F10 tumors implanted subcutaneously were established (Fig. 5i). Treatment with the $\operatorname{imNA}_{\alpha \mathrm{CSF} 1 \mathrm{R}} \& \alpha \mathrm{CD} 47$ via an intratumoral injection at a predetermined dose showed a significant tumor-inhibition effect compared with Free ${ }_{\alpha C S F 1 R}$ \& ${ }_{\alpha C D 47}$ and $\mathrm{NP}_{\alpha \mathrm{CSF} 1 \mathrm{R}} \& \mathrm{NP}_{\alpha \mathrm{CD} 47}$ (Fig. 5j, k). As expected, imNA $_{\alpha C S F 1 R} \& a_{\alpha C D} 47$ administration also extended the survival time of tumor-bearing mice (Supplementary Fig. 30). Flow cytometry analysis and TAM-depleting experiments showed that the excellent tumor inhibitory effect was attributed to the improved phagocytosis of tumor cells by TAMs (Supplementary Figs. 31 and 32). Surprisingly, imNA $\mathrm{aCSF}_{\mathrm{R}}$ \& aCD47 could also reverse the immunosuppressive tumor microenvironment, as assessed by decreased M2 macrophages and increased M1 macrophages, T cells, and NK cells (Supplementary Fig. 33).

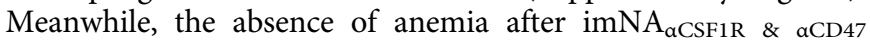
administration validated the biological safety of our treatment regimens (Supplementary Fig. 34). Collectively, these results confirmed that aFc-NP could be a versatile and facile antibody immobilization platform; and multiple imNAs could be obtained by mixing aFc-NP with two types of well-chosen mAbs, and substantially improving the therapeutic efficacy of original mAbs.

\section{Discussion}

Immunotherapy has attracted much more attention than traditional cancer treatment strategies (surgery, chemotherapy, radiation, and molecularly targeted therapy) in recent years, especially due to the remarkable success of immune checkpoint blockade in patients with a wide variety of malignancies ${ }^{39}$. As the predominant modality of cancer immunotherapy, immunomodulatory monoclonal antibodies (mAbs), including mAbs targeting co-inhibitory or co-stimulatory ligands/receptors of T cells and NK cells (e.g., PDL1, PD1, CTLA4, OX40, 4-1BB, NKG2D, TIGIT) ${ }^{3,40}$, mAbs re-educating or depleting TAMs (e.g., CSF1R, CCR 2$)^{7}$, and mAbs promoting the phagocytosis of macrophages and dendritic cells (CD47, SIRPa $)^{41,42}$, may revolutionize the cancer treatment paradigm in the future. However, the clinical benefit of immunomodulatory mAbs is limited to a minority of patients due to innate and/or acquired resistance as well as their inevitable adverse effects ${ }^{43}$. Several approaches have been developed to improve the antitumor efficacies of immunomodulatory mAbs: (I) combining two types of mAbs with distinct mechanisms of action ${ }^{44}$; (II) combining mAbs with other treatment modalities 45 ; and (III) increasing the tumor accumulation of mAbs via antibody modification or various delivery systems ${ }^{45-47}$. Undoubtedly, these approaches can improve the antitumor activities of mAbs to a certain extent; meanwhile, novel strategies that can broaden the clinical utility of immunomodulatory mAbs are still urgently needed.

Bispecific antibodies (bsAbs), which comprise two antigenrecognizing elements that are capable of simultaneously binding two distinct targets, are emerging as a promising format of cancer immunotherapy. Blinatumomab $(\mathrm{CD} 3 \times \mathrm{B}$ lymphocyte antigen CD19) has been utilized to treat B-cell acute lymphocytic leukemia with impressive clinical results, and more than 50 bsAbs are currently being evaluated for the treatment of hematological and solid tumors ${ }^{48}$. The unique and highly advantageous feature of bsAbs over monospecific antibodies is the capability of triggering contact between effector cells and tumor cells and consequently enhancing cytotoxicity, which is unachievable for the mixture of monospecific antibodies. We speculated that integrating the features of mAbs (immunomodulating function of immune cells) and bsAbs (facilitating co-engagement of immune cells and tumor cells) into one system could dramatically boost immunotherapy, and we proposed that nanoparticle immobilizing two types of mAbs targeting effector cells and tumor cells could be such a system.

Emerging research has indicated that engineering multi mAbs onto nanoparticles may promote the interaction between effector cells and tumor cells, yet the underlying mechanism has not been fully elucidated ${ }^{49}$. More importantly, previously reported antibody immobilization approaches mainly rely on chemical reactions through primary amine groups, thiol groups, or sugar chains. However, these processes are difficult to control due to the high molecular weight and the multivalent properties of antibodies and nanoparticles, and they may also potentially hurt the valency of antibodies, limiting its clinical translation. Considering that all clinically approved mAbs for cancer immunotherapy are IgG isotypes comprising well-conserved Fc fragments, we proposed that nanoscale formulations decorated with anti-IgG (Fc specific) antibodies $(\alpha \mathrm{Fc})$ could be a versatile antibody immobilization platform ( $\alpha \mathrm{Fc}-\mathrm{NP})$, and it could theoretically immobilize any $\mathrm{mAbs}$ through $\mathrm{Fc}$-specific recognition and interactions, which is utterly different from rough and complex chemical reactions. We successfully constructed $\mathrm{aFc}-\mathrm{NP}$ via oriented conjugation of $\alpha \mathrm{Fc}$ onto nanoparticles and showed that $\mathrm{aFc}-\mathrm{NP}$ can efficiently immobilize multiple therapeutic mAbs through gentle mixing. Disruption of antigen-binding capability (e.g., blockage of antigen-binding sites, destruction of antibody structure) is an intractable problem always encountered in antibody immobilization $^{29}$. Encouragingly, without any chemical modification, mAbs can be immobilized by $\alpha \mathrm{Fc}-\mathrm{NP}$ through noncovalent interactions. Additionally, the $\mathrm{F}(\mathrm{ab})_{2}$ fragments of therapeutic mAbs face outward after immobilization, and their antigen-binding capacities are preserved to the greatest extent.

Be parallel to the facile construction process was the impressive antitumor efficacy of $\alpha \mathrm{Fc}-\mathrm{NP}$ immobilizing two types of mAbs against effector cells and tumor cells (referred to as immunomodulating nano-adaptors, imNAs). We selected aPDL1 and

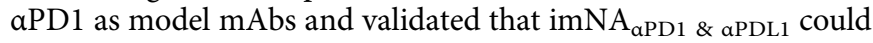
integrate the functions of $\mathrm{mAbs}$ and bsAbs. First, immobilized aPDL1 and $\alpha \mathrm{PD} 1$ retained their intrinsic immunomodulatory properties and could reinforce the cytotoxicity of $\mathrm{CD}^{+} \mathrm{T}$ cells against tumor cells. Second, as imNA $\mathrm{\alpha PD1}_{\alpha}$ aPDL1 integrated aPDL1 against tumor cells and aPD1 against T cells, it could associate with both cells simultaneously and act as an 'adaptor' to tightly connect them. In addition, considering that multiple mAbs were integrated by single $\alpha \mathrm{Fc}-\mathrm{NP}$ (known as multivalence), we

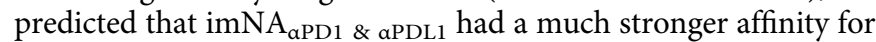


both cells compared with conventional bsAbs. Their amplified antitumor efficacy over the mixture of $\alpha \mathrm{PDL} 1$ and $\alpha \mathrm{PD} 1$ in vitro and in vivo indicated that $\mathrm{imNA}_{\mathrm{aPD} 1} \&$ aPDL1- facilitated $\mathrm{T}$ cell/ tumor cell interactions worked synergistically with the modulation of T-cell function by aPD1 \& aPDL1. The general applicability of $\alpha \mathrm{Fc}-\mathrm{NP}$ and therapeutic superiority of imNAs were further validated in natural killer cell- and macrophage-mediated antitumor immune responses using murine subcutaneous melanoma, orthotopic breast tumor, and lung metastasis models, encouraging the clinical translation of $\alpha \mathrm{Fc}-\mathrm{NP}$ and imNAs. $\alpha \mathrm{Fc}-$ NP can potentially serve as a universal 'adjuvant' to various FDA (Food and Drug Administration)-approved $\mathrm{mAbs}$ and those being evaluated in clinical trials, facile mixing is expected to significantly improve the antitumor efficacies of mAbs. Additionally, although the present work focused on cancer immunotherapy, the versatile nanoplatform we reported here could be extended to the fields of targeted nanomedicine, disease diagnosis, and antibody engineering. For instance, bi-, tri-, or multi-specific antibodies can be constructed by mixing $\alpha \mathrm{Fc}-\mathrm{NP}$ with certain mAbs instead of a sophisticated molecular design and genetic engineering ${ }^{50}$.

\begin{abstract}
Methods
Materials. Amino-functionalized polystyrene nanoparticles (NP) $(\sim 120 \mathrm{~nm}$ in diameter) were purchased from Shanghai Macklin Biochemical Co., Ltd. Sodium periodate $\left(\mathrm{NaIO}_{4}\right)$ was obtained from Shanghai Aladdin Bio-Chem Technology Co., Ltd. Sodium borohydride $\left(\mathrm{NaBH}_{4}\right)$ was obtained from Energy Chemical (Shanghai, China). The goat anti-rat IgG ( $\mathrm{Fc}$ specific) antibody $(\mathrm{aFc})$ was obtained from Rockland Immunochemicals Inc. The InVivoPlus anti-mouse PD1 antibody (Clone: 29 F.1A12), anti-mouse PDL1 antibody (Clone: 10 F.9G2), anti-mouse KLRG1 antibody (Clone: 2F1), anti-mouse CD47 antibody (Clone: MIAP410), anti-mouse CSF1R antibody (Clone: AFS98) and rat IgG2a isotype control (antitrinitrophenol, Clone: 2A3) were obtained from Bio X Cell. The fluorochromelabeled antibodies for flow cytometry detection were purchased from BioLegend. The PKH26 Red Fluorescent Cell Linker Mini Kit, collagenase type I, hyaluronidase, and DNase I were obtained from Sigma-Aldrich. The CellTrace ${ }^{m i}$ Blue Cell Proliferation Kit, CellTrace ${ }^{\mathrm{ma}}$ CFSE Cell Proliferation Kit, Alexa Fluor ${ }^{\circledR} 647$ dye (AF647), and Alexa Fluor ${ }^{\circledR} 750$ dye (AF750) was obtained from Thermo Fisher Scientific. Recombinant mouse PD1 and PDL1 proteins (rmPD1 and rmPDL1) were purchased from Sino Biological, Inc. Sulfo-Cyanine5 NHS ester and fluorescein isothiocyanate (FITC) were purchased from J\&K Scientific. Cell and antibody labeling were performed according to the manufacturers' protocols.
\end{abstract}

Cell lines and animals. The B16-F10 murine melanoma cells and 4T1 murine mammary carcinoma cells were obtained from the American Type Culture Collection (ATCC). B16-F10 cells expressing membrane-bound chicken ovalbumin (B16-F10-OVA), B16-F10 cells expressing mCherry (B16-F10-mCherry), 4T1 cell expressing GFP, 4T1 cells expressing firefly luciferase (4T1-fLuc) were constructed by transfecting OVA-, mCherry-, GFP- or fluc-encoding lentiviral vectors (Vectorbuilder) into B16-F10 or 4T1 cells. Cells were maintained in Dulbecco's modified Eagle medium (B16-F10 cells) or RPMI-1640 medium (4T1 cells) supplemented with $10 \%$ fetal bovine serum (FBS, Gibco) and $1 \%$ penicillin/ streptomycin (Invitrogen) in a humidified atmosphere containing $5 \% \mathrm{CO}_{2}$ at $37^{\circ} \mathrm{C}$. Transduced cells were grown in medium containing the selection agent puromycin at a concentration of $0.25 \mu \mathrm{g} / \mathrm{mL}$. Primary $\mathrm{CD}^{+}{ }^{+} \mathrm{T}$ cells were isolated from the spleens of C57BL/6 or OT-1 transgenic mice using a CD8a (Ly2) microbeads isolation kit (Miltenyi Biotec.), and then cultured in RPMI-1640 medium supplemented with $10 \%$ FBS, $1 \%$ penicillin/streptomycin, 1\% GlutaMAX (Life Technologies), $10 \mathrm{mM}$ HEPES (Life Technologies), $1 \mathrm{mM}$ sodium pyruvate (Life Technologies), $55 \mu \mathrm{M}$ 2-mercaptoethanol (Life Technologies) and $10 \mathrm{ng} / \mathrm{mL}$ IL-2 (Peprotech). All cells were confirmed to be Mycoplasma-free using Hoechst DNA staining and agar culture methods.

Male C57BL/6 mice and female BALB/c mice were purchased from Hunan Silaikejingda Laboratory Animal Technology Co. Ltd. OT-I (C57BL/6-Tg (TcraTcrb) $1100 \mathrm{Mjb} / \mathrm{J}$ ) TCR transgenic mice were a generous gift from Professor Tian-Meng Sun from Jilin University. All mice were maintained at the animal facility of South China University of Technology (SCUT) in a specific pathogenfree (SPF) environment with controlled temperature $\left(\sim 22^{\circ} \mathrm{C}\right)$ and humidity $(50 \pm$ $15 \%)$ under $12 \mathrm{~h}$ light/dark cycle. Mice aged 6-8 weeks were used for experiments. All animal experiments were approved by the Animal Care and Use Committee at SCUT, and every effort was made to minimize suffering from experiments.

$\boldsymbol{\alpha F c}$ oxidation and $\boldsymbol{\alpha F c}$ conjugation. The oxidation of carbohydrate residues on the $\mathrm{Fc}$ portion of $\alpha \mathrm{Fc}$ was performed by dissolving $1 \mathrm{mg} / \mathrm{mL} \alpha \mathrm{Fc}$ in $50 \mathrm{mM}$ acetate buffer ( $\mathrm{pH} 4.2)$ containing sodium periodate $\left(\mathrm{NaIO}_{4}, 10 \mathrm{mM}\right)$ for $2 \mathrm{~h}$ at $4{ }^{\circ} \mathrm{C}$, and oxidized $\alpha \mathrm{Fc}$ was recovered using Amicon ${ }^{\circledR}$ Ultra Centrifugal Filters (MWCO $100 \mathrm{kDa}$, Merck Millipore) (centrifugation at $9,000 \times g$ for $5 \mathrm{~min}$ ). The generation of aldehydes was detected by Purpald ${ }^{\oplus}$ (4-amino-3-hydrazino-5-mercapto-1,2,4 triazole, Sigma). Then, oxidized $\alpha \mathrm{Fc}(0.1 \mathrm{mg} / \mathrm{mL})$ was mixed with aminated NP at predefined mass ratios, and the reaction between primary amine and aldehyde groups was performed for $12 \mathrm{~h}$ at $4{ }^{\circ} \mathrm{C}$ with gentle stirring. Finally, sodium borohydride $\left(\mathrm{NaBH}_{4}\right.$, Energy Chemical) was added to the mixtures and incubated for another $45 \mathrm{~min}$ to reduce the Schiff base intermediates and generate stable covalent linkages between $\mathrm{NP}$ and $\alpha \mathrm{Fc}$. The fabricated formulations ( $\alpha \mathrm{Fc}-\mathrm{NP})$ were collected by centrifugation, and the unbound $\alpha \mathrm{Fc}$ in supernatants was examined by enzymelinked immunosorbent assay (ELISA) (goat IgG ELISA Kit, Alpha Diagnostic International) and ultra-performance liquid chromatography (UPLC). The binding efficacy $(\mathrm{BE})$ of $\alpha \mathrm{Fc}$ was calculated using the following formula: $\mathrm{BE}=(\mathrm{A}-\mathrm{B}) / \mathrm{A}$, where $\mathrm{A}$ is the feeding amount of $\alpha \mathrm{Fc}$ and $\mathrm{B}$ is the $\alpha \mathrm{Fc}$ in the supernatant.

To measure the size distribution, $\mathrm{NP}$ and $\alpha \mathrm{Fc}-\mathrm{NP}$ were diluted in a $5 \%$ glucose solution $(1 \mathrm{mg} / \mathrm{mL})$ and characterized using a Zetasizer Nano ZS instrument (Malvern, Inc.). For the morphology examination, NP and $\alpha \mathrm{Fc}-\mathrm{NP}$ were drop cast onto silicon wafers and imaged using a field emission scanning electron microscope (Merlin Compact, Zeiss, Germany)

The method by which NP binds $\alpha \mathrm{Fc}$ was identified by reducing SDS-PAGE (sodium dodecyl sulfate-polyacrylamide gel electrophoresis). Briefly, free $\alpha \mathrm{Fc}$ or $\alpha F c-N P$ diluted in PBS (phosphate-buffered saline) was mixed with SDS-PAGE sample loading buffer $(5 \times)$ (GeneCopoeia) and heated for $10 \mathrm{~min}$ at $99^{\circ} \mathrm{C}$. The reducing agent $\beta$-mercaptoethanol in sample loading buffer breaks the intrachain disulfide bonds of $\alpha \mathrm{Fc}$ and separates heavy chains and light chains. Then, the mixtures $(20 \mu \mathrm{L}, 500 \mu \mathrm{g} / \mathrm{mL})$ were loaded onto polyacrylamide gels and run at $90 \mathrm{~V}$ for approximately $120 \mathrm{~min}$, followed by staining with Coomassie Blue (Beijing Solarbio Science \& Technology Co., Ltd). The bands of heavy chains $(\sim 50 \mathrm{kDa})$ and light chains $(\sim 25 \mathrm{kDa})$ were imaged with Typhoon Gel and Blot Imaging Systems (GE Healthcare) after destaining.

Construction and characterization of $\alpha \mathrm{Fc}-\mathrm{NP} / \mathrm{mAbs}$. $\alpha \mathrm{PD} 1$ was mixed with $\alpha \mathrm{Fc}-\mathrm{NP}$ at the $\alpha \mathrm{PD} 1 / \alpha \mathrm{Fc}$ ratio of $1: 1(\mathrm{w} / \mathrm{w})$ and incubated for $12 \mathrm{~h}$ at $4{ }^{\circ} \mathrm{C}$, and the

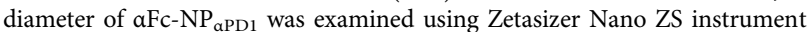
(Malvern, Inc.). To confirm that $\alpha \mathrm{PD} 1$ was immobilized onto $\alpha \mathrm{Fc}-\mathrm{NP}$ via $\mathrm{Fc}$ specific recognition, the IgG isotype control or $\alpha \mathrm{Fc}$ was conjugated to AF750labeled NP, followed by incubation with AF647-labeled aPD1. The mixtures (IgG$\mathrm{NP}_{\alpha \mathrm{aP} 1}$ or $\left.\alpha \mathrm{Fc}-\mathrm{NP}_{\alpha \mathrm{PD} 1}\right)$ were dropped on the coverslip and precipitated for $10 \mathrm{~min}$, and images were acquired using a SRiS STORM Super-resolution System (NanoBioImaging, Ltd.). SRiS is equipped with $647 \mathrm{~nm}$ and $750 \mathrm{~nm}$ super-resolution compatible laser excitation, which enables industry-leading two-color simultaneous STORM acquisition.

To confirm that $\alpha \mathrm{Fc}-\mathrm{NP}$ could integrate two types of mAbs simultaneously, PerCP-Cy5.5-conjugated aPDL1 and FITC-conjugated aPD1 (BioLegend) were incubated alone or in combination with $\alpha \mathrm{Fc}-\mathrm{NP}$ for $12 \mathrm{~h}$ and then subjected to high-sensitivity nanoflow cytometry (HSFCM, NANOFCM CO., LTD). The HSFCM instrument was equipped with three single-photon-counting avalanche photodiode (APD) detectors for the simultaneous detection of side scattering (SSC) and two-color fluorescence. The acquired data were analyzed by FlowJo v10 (Tree Star).

To detect stability, $a \mathrm{Fc}-\mathrm{NP}_{\mathrm{aPD} 1}$ was incubated with $5 \%$ glucose for $48 \mathrm{~h}$ and the diameter variation was examined using a Zetasizer Nano ZS instrument (Malvern Inc.). To investigate the stability of $\alpha \mathrm{Fc}-\mathrm{NP}_{\alpha \mathrm{PD} 1}$ in the presence of IgG from the same or different host species, aFc-NP integrating PerCP-Cy5.5-labeled PDL1 $\left(\alpha \mathrm{Fc}-\mathrm{NP}_{\mathrm{PP}-\mathrm{Cy5} 5 \text { - }-\mathrm{\alpha PDL} 1}\right)$ was incubated with the rat IgG control and mouse IgG control, and the concentrations of PerCP-Cy5.5- $\alpha$ PDL1, rat IgG control, and mouse IgG were $10 \mu \mathrm{g} / \mathrm{mL}$. The percentage of PerCP-Cy5.5 ${ }^{+} \alpha \mathrm{Fc}-\mathrm{NP}$ PP-Cy5.5-aPD1 after $12 \mathrm{~h}$ and $24 \mathrm{~h}$ incubation was examined by nanoflow cytometry (HSFCM, NANOFCM CO., LTD).

$\alpha P D 1$ and $\alpha P D L 1$ binding efficiency. $\alpha$ PD1 or $\alpha$ PDL1 were mixed with $\alpha \mathrm{Fc}-\mathrm{NP}$ (the mass ratio of $\alpha \mathrm{PD} 1$ and $\alpha \mathrm{PDL} 1$ to $\alpha \mathrm{Fc}$ was $1: 1$ ) and incubated at $4{ }^{\circ} \mathrm{C}$ for a predetermined time $(1,2,4,8,12,24 \mathrm{~h})$. The solution was centrifuged at $20,000 \times g$ for $60 \mathrm{~min}$, and the unbound aPD1 and aPDL1 in the supernatant were examined by ELISA. Briefly, 96-well plates (Corning) were coated with $100 \mu \mathrm{L}(5 \mu \mathrm{g} / \mathrm{mL})$ rmPD1 or rmPDL1 for $2 \mathrm{~h}$ at $37^{\circ} \mathrm{C}$, followed by blocking with $2 \%$ BSA (bovine serum albumin, Sigma) in PBST $\left(1 \times\right.$ PBS containing $0.1 \%$ Tween $\left.^{\circledast} 20\right)$ for $1 \mathrm{~h}$ at room temperature (RT). Samples were then incubated with free aPD1 or aPDL1 (a series of concentration gradients) or the collected supernatant for $1 \mathrm{~h}$ at RT. After washing, $100 \mu \mathrm{L}(1 \mu \mathrm{g} / \mathrm{mL}) \mathrm{HRP}$-conjugated goat anti-rat IgG antibody (Sino Biological, Inc.) was added as the detection antibody and incubated for $1 \mathrm{~h}$ at RT, followed by the addition of $100 \mu \mathrm{L}$ of 3,3',5,5'-tetramethylbenzidine (TMB, Abcam). After another $10 \mathrm{~min}$ incubation, a $450 \mathrm{~nm}$ stop solution for the TMB substrate was added, and the absorbance intensity in each well was detected using an 800 TS microplate reader (Biotek).

To examine whether aFc-NP could bind two types of mAbs in a controlled manner, $\alpha \mathrm{PD} 1$ and $\alpha \mathrm{PDL} 1$ (the mass ratios of $\alpha \mathrm{PD} 1$ to $\alpha \mathrm{PD} 1$ ranged from $1 / 3$ to 3 ) were incubated with $\alpha \mathrm{Fc}-\mathrm{NP}$ (the mass ratio of $\alpha \mathrm{PD} 1 \& \alpha \mathrm{PDL} 1$ to $\alpha \mathrm{Fc}$ was 1:1) for $12 \mathrm{~h}$ at $4{ }^{\circ} \mathrm{C}$. The solution was centrifuged at $20,000 \times g$ for $60 \mathrm{~min}$, and the 
unbound $\alpha \mathrm{PD} 1$ and $\alpha \mathrm{PDL} 1$ in the supernatant were examined by ELISA as indicated above.

Antigen-binding capability. ELISA plates (Corning) were coated with $100 \mu \mathrm{L}$ $(5 \mu \mathrm{g} / \mathrm{mL})$ of rmPD1 or rmPDL1 for $2 \mathrm{~h}$ at $37^{\circ} \mathrm{C}$, followed by blocking with $2 \% \mathrm{BSA}$ in PBST for $1 \mathrm{~h}$ at RT. A series of concentrations of free mAbs ( $\mathrm{APD} 1$ or $\mathrm{aPDL1}$ ) or NP-immobilized $m A b s\left(\alpha F c-N_{\alpha P D 1}\right.$ or $\left.\alpha \mathrm{Fc}-\mathrm{NP}_{\alpha \mathrm{aPL} 1}\right)$ were added and incubated for $1 \mathrm{~h}$ at RT. The attached antibodies were examined as indicated above. The dissociation constant $\mathrm{K}_{\mathrm{D}}$ was obtained by plotting normalized absorbance values versus the concentrations of $\alpha \mathrm{PD} 1$ or aPDL1 using PRISM software (GraphPad).

Stimulation of tumor cells and CD8 ${ }^{+} \mathbf{T}$ cells in vitro. B16-F10 or B16-F10mCherry cells were stimulated with IFN- $\gamma(20 \mathrm{ng} / \mathrm{ml}$, Peprotech) for $24 \mathrm{~h}$. Murine spleens were carefully removed and washed three times with sterile PBS on ice. The spleens were gently fragmented between glass microscope slides and the splenocyte suspension was filtered through a 40- $\mu \mathrm{m}$ nylon mesh. Red blood cells were removed using ACK (Ammonium-Chloride-Potassium) Lysing Buffer (BioLegend), the splenocytes were then resuspended in magnetic-activated cell sorting (MACS) buffer and CD8 ${ }^{+} \mathrm{T}$ cells were isolated via a CD8a (Ly2) microbeadsIsolation Kit (Miltenyi Biotec.). For $\mathrm{T}$ cell stimulation, isolated $\mathrm{CD} 8^{+} \mathrm{T}$ cells were incubated with plate-bound anti-CD3 antibodies $(5 \mu \mathrm{g} / \mathrm{mL}$, BioLegend) and soluble anti-CD28 antibodies $(5 \mu \mathrm{g} / \mathrm{mL}$, BioLegend) for $48 \mathrm{~h}$. PD1 or PDL1 expression after stimulation was assessed using a BD FACSCelesta ${ }^{\text {tw }}$ flow cytometer (BD Biosciences).

Association between cells and imNA QPD1 $_{\text {\& } \alpha \text { PDL1. }}$ Briefly, $1.0 \times 10^{5}$ stimulated B16-F10 cells or $\mathrm{CD} 8{ }^{+} \mathrm{T}$ cells were seeded into 24 -well plates and incubated overnight, followed by the addition of $\alpha \mathrm{Fc}$-NP immobilizing IgG control $(\alpha \mathrm{Fc}-$ $\mathrm{NP}_{\mathrm{IgG}}$ ) or $\alpha \mathrm{Fc}-\mathrm{NP}$ immobilizing $\alpha \mathrm{PD} 1$ and $\alpha \mathrm{PDL} 1\left(\mathrm{imNA}_{\alpha \mathrm{PD} 1} \& \mathrm{\alpha PDL1}\right)$. NPs were labeled with FITC, and the concentration of $\alpha \mathrm{PD} 1$ and $\alpha \mathrm{PDL} 1$ was $10 \mu \mathrm{g} / \mathrm{mL}$. After timed intervals, cells were washed three times with ice-cold PBS, and the mean fluorescence intensity (MFI) of FITC from B16-F10 cells or $\mathrm{CD}^{+} \mathrm{T}$ cells was detected on a BD FACSCelesta ${ }^{\text {Tw }}$ flow cytometer (BD Biosciences). For the distinction between internalized and surface-bound $\alpha \mathrm{Fc}^{-\mathrm{NP}_{\mathrm{IgG}}}$ or imNA $\mathrm{APD}_{\alpha \text { \& }}$ aPDL1 , trypan blue $(0.4 \%$, Thermo Fisher Scientific), which has been demonstrated to quench the fluorescence of FITC-labeled compounds when in close contact with them, was used to quench surface-bound fluorescence and added to the cell suspension before FACS acquisition. The surface-bound fluorescence (SBF) was calculated using the following equation: $\mathrm{SBF}=\mathrm{A}-\mathrm{B}$, where $\mathrm{A}$ and $\mathrm{B}$ represent the MFI without or with trypan blue quenching, respectively.

To directly visualize the association between cells and imNA $\mathrm{aPD}_{\mathrm{aP}}$ \& aPDL1, $\mathrm{B} 16-$ F10 cells or $\mathrm{CD}^{+} \mathrm{T}$ cells were labeled with red fluorescent dye using the PKH26 Red Fluorescent Cell Linker Kit (Sigma) according to the manufacturer's protocol

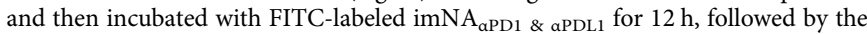
observation using a laser scanning confocal microscope (Zeiss).

\section{imNA $A_{\alpha P D 1} \& \alpha P D L 1$ facilitates the interaction between tumor cells and CD8 ${ }^{+}$} T cells. B16-F10-mCherry cells and isolated $\mathrm{CD} 8^{+} \mathrm{T}$ cells were stimulated as indicated above. B16-F10-mCherry cells were seeded into $\mathrm{Nunc}^{\mathrm{m}}$ glass-bottom dishes (Thermo Fisher Scientific) at a density of $5.0 \times 10^{3}$ cells per dish, and $5.0 \times$ $10^{4} \mathrm{CFSE}$-labeled CD8 ${ }^{+} \mathrm{T}$ cells were added $4 \mathrm{~h}$ later. Cocultured cells were treated with IgG control $(20 \mu \mathrm{g} / \mathrm{mL})$, Free $\alpha_{\alpha P 1} \& \alpha \operatorname{aPDL}_{1}, \mathrm{NP}_{\alpha \mathrm{PDD} 1} \& \mathrm{NP}_{\alpha \mathrm{PDL} 1}$ or $\mathrm{imNA}_{\alpha \mathrm{PD} 1}$ \& aPDL1; the concentration of $\alpha$ PD1 and $\alpha$ PDL1 was $10 \mu \mathrm{g} / \mathrm{mL}$. After an additional $8 \mathrm{~h}$ incubation, suspended $\mathrm{CD}^{+}{ }^{+} \mathrm{T}$ cells were removed, and $\mathrm{T}$ cell-tumor cell conjugations were visualized under a Zeiss LSM880 laser scanning confocal microscope. The conjugation rates, measured as the percentage of B16-F10 cells conjugating one or more $\mathrm{CD} 8^{+} \mathrm{T}$ cells, were determined manually by observing red/green contact in multiple nonoverlapping images using ImageJ v1.47 (National Institutes of Health, USA).

Detection of IFN- $\gamma$, granzyme B, and perforin via ELISA. A total of $5.0 \times 10^{3}$ B16-F10 cells and $5.0 \times 10^{4}$ stimulated CD8 ${ }^{+} \mathrm{T}$ cells were seeded into 96-well plates (Corning) and incubated overnight. Cocultured cells were treated with $\operatorname{IgG}$

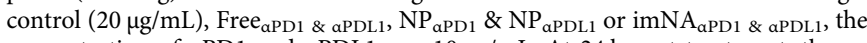
concentration of $\mathrm{QPD} 1$ and $\alpha \mathrm{PDL} 1$ was $10 \mu \mathrm{g} / \mathrm{mL}$. At $24 \mathrm{~h}$ post-treatment, the supernatant was collected, and the modulators of T cell-mediated cytotoxicity in the supernatant were quantified via Mouse IFN- $\gamma$ ELISA Kit (Dakewe Biotech), Mouse Granzyme B ELISA Kit (Abcam), and Mouse Perforin 1 ELISA Kit (Abbexa) according to the manufacturer's protocol.

In vitro apoptosis assay. To examine the cytotoxicity of nonantigen-specific $\mathrm{T}$ cells, B16-F10-mCherry cells were seeded into CellCarrierUltra ULA 96-well microplates (PerkinElmer) at a density of $5.0 \times 10^{3}$ cells per well and allowed to adhere for $12 \mathrm{~h}$, and $5.0 \times 10^{4}$ stimulated $\mathrm{CD} 8^{+} \mathrm{T}$ cells labeled with CellTrace Blue were then added. Cocultured cells were treated with IgG control $(20 \mu \mathrm{g} / \mathrm{mL})$,

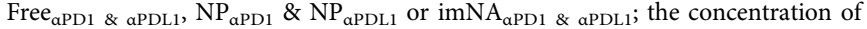
$\alpha \mathrm{PD} 1$ and $\alpha \mathrm{PDL} 1$ was $10 \mu \mathrm{g} / \mathrm{mL}$. FITC conjugated recombinant Annexin V (Annexin V-FITC, Thermo Fisher Scientific) was added to the medium (final concentration was $1 \mu \mathrm{g} / \mathrm{mL}$ ) to detect apoptotic cells. The plates were incubated at $37^{\circ} \mathrm{C}$ and $5 \% \mathrm{CO}_{2}$, and images of cocultured cells were continuously acquired every $45 \mathrm{~min}$ using the Operetta CLS ${ }^{\mathrm{mi}}$ High-Content Analysis System (PerkinElmer) for $48 \mathrm{~h}$. The viability of B16-F10 cells was evaluated using Harmony high-content analysis software based on cellular phenotypes and fluorescence distribution parameters.

To examine the cytotoxicity of antigen-specific $\mathrm{T}$ cells, $\mathrm{CD}^{+} \mathrm{T}$ cells isolated from OT-1 transgenic mice were cultured and stimulated with anti-CD3/28 antibodies $(5 \mu \mathrm{g} / \mathrm{mL})$ for $24 \mathrm{~h}$. CD8 ${ }^{+} \mathrm{T}$ cells were then cocultured with Hoechst 33342 (H33342)-labeled B16-F10-OVA cells at ratios of 5:1 or 10:1 in 100 $\mu \mathrm{L}$ media in the presence of the IgG control antibody $(20 \mu \mathrm{g} / \mathrm{mL})$, Free ${ }_{\alpha \mathrm{PD} 1} \&$ aPDL1, $\mathrm{NP}_{\alpha \mathrm{aPD} 1}$ $\& \mathrm{NP}_{\alpha \mathrm{PDL} 1}$ or $\mathrm{imNA}_{\mathrm{aPD} 1} \& \alpha \mathrm{\alpha PD} 1$ for $48 \mathrm{~h}$, and the concentration of $\alpha \mathrm{PD} 1$ and aPDL1 was $10 \mu \mathrm{g} / \mathrm{mL}$. After co-incubation, the amount of $\mathrm{H} 33342$ in the supernatant and adherent cells was examined using an Infinite ${ }^{\circledast} 200$ PRO microplate plate reader, and cell viability was calculated using the following formula: cell viability $=(\mathrm{A}-\mathrm{B}) / \mathrm{A}$, where $\mathrm{A}$ is the total amount of $\mathrm{H} 33342$, and $\mathrm{B}$ is the $\mathrm{H} 33342$ in the supernatant.

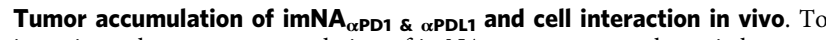
investigate the tumor accumulation of $\mathrm{imNA}_{\mathrm{\alpha PD} 1}$ \& $\alpha \mathrm{PDL}$, orthotopic breast tumor models were established by injecting $5.0 \times 10^{5} 4 \mathrm{~T} 1$ cells into the mammary fat pad of female BALB/c mice (6-8 weeks old). Mice were randomly divided into three groups when the tumor volume reached approximately $300 \mathrm{~mm}^{3}$, followed by intravenous administration of PBS, Free $\mathrm{aPD}_{\text {\& } ~ \& ~ a P D L 1}$ or imNA $\mathrm{aPD}_{\text {\& }}$ \& aPDL1; $\alpha \mathrm{PD} 1$ and $a$ PDL1 were labeled with Cy5 dye (Thermo Fisher Scientific) and the injection dose of Cy5-labeled aPD1 \& aPDL1 was $5.0 \mathrm{mg}$ per $\mathrm{kg}$ mouse body weight. Mice were sacrificed at predetermined time intervals, and tumor tissues were harvested for fluorescence imaging using the In-Vivo Xtreme II imaging system (Bruker). The acquired images were analyzed using Molecular Imaging Software (Bruker). Next, tumor tissues were fixed in $4 \%$ paraformaldehyde (Sigma) overnight at $4{ }^{\circ} \mathrm{C}$, immersed in 30\% sucrose solution overnight, and then sectioned into $10 \mu \mathrm{m}$ pieces and stained with DAPI (4',6-diamidino-2-phenylindole) for confocal microscopy observation (Zeiss).

To validated whether imNA $\mathrm{aPD1}_{\mathrm{\alpha} \text { aPDL1 }}$ could enhance the interaction between $\mathrm{CD}^{+} \mathrm{T}$ cells and tumor cells, mice bearing 4T1-GFP tumors were treated with

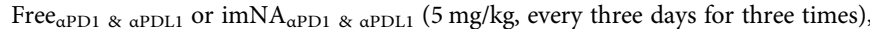
$24 \mathrm{~h}$ post-final injection, tumor tissues were harvested and stained with anti-CD8a antibody and Alexa Fluor ${ }^{\circledast} 568$ labeled goat anti-rabbit IgG H\&L antibody, followed by confocal observation.

Anti-tumor study. For the subcutaneous melanoma model, $2.0 \times 10^{5} \mathrm{~B} 16-\mathrm{F} 10$ cells in $100 \mu \mathrm{L}$ of PBS were subcutaneously inoculated into the right flank of the 6-8week-old female C57BL/6 mice. For the orthotopic breast tumor model, $5.0 \times 10^{5}$ $4 \mathrm{~T} 1$ cells were injected into the mammary fat pads of female $\mathrm{BALB} / \mathrm{c}$ mice. When the tumor volumes were approximately $50 \mathrm{~mm}^{3}$, mice were randomly divided into four groups (6 or 10 mice per group) and intravenously or intratumorally administered the corresponding formulations on a schedule of an injection every 3 days for a total of three injections (q3dx3). For imNA $\mathrm{aPD} 1 \&$ aPDL1 , the doses of aPD1 and $\alpha$ PDL1 were $2.5 \mathrm{mg} / \mathrm{kg}$, while for $\mathrm{imNA}_{\mathrm{KLRG1}} \& \alpha \mathrm{\alpha PL1}$ and $\mathrm{imNA} \mathrm{ACSF1R}_{\alpha}$ aCD47, the doses of $\alpha$ KLRG1, aPDL1, aCSFR1 and $\mathrm{aCD} 47$ were $1.5 \mathrm{mg} / \mathrm{kg}$. Tumor volumes were monitored by measuring the perpendicular diameter with a caliper, and the estimated volume was calculated based on the following equation: $V=L \times$ $W^{2} \times 1 / 2$ ( $V$, volume; $L$, length; $W$, width of tumor). Body weights were monitored every three days. The survival rates were expressed using Kaplan-Meier survival analysis.

For the lung metastasis model, $7.5 \times 10^{4} 4 \mathrm{~T} 1$-fLuc cells or $5.0 \times 10^{4} \mathrm{~B} 16$-F10 cells were administered via the tail vein into female $\mathrm{BALB} / \mathrm{c}$ or $\mathrm{C} 57 \mathrm{BL} / 6$ mice, and treatments were initiated the following day as indicated above. For the bioluminescence observation, mice were intraperitoneally administered $3 \mathrm{mg}$ of the D-luciferin (Dalian Meilun) in $200 \mu \mathrm{L}$ of PBS, followed by the observation via InVivo Xtreme II imaging system (Bruker). On day $16, \mathrm{BALB} / \mathrm{c}$ mice were sacrificed and the lungs were separated for bioluminescence imaging after soaking in the Dluciferin solution. On day 20, C57BL/6 mice were sacrificed, and the lungs were separated. The metastatic foci in lung tissues were counted and recorded. Then, lung tissues were fixed in $4 \%$ paraformaldehyde (Sigma) and embedded in paraffin; the paraffin-embedded lungs were cut and stained with hematoxylin-eosin for immunohistochemical analysis.

Flow cytometry analysis. Tumor tissues were harvested, minced, and incubated with RPMI-1640 medium containing $10 \%$ FBS $(\mathrm{v} / \mathrm{v})$, collagenase type I $(1 \mathrm{mg} / \mathrm{mL})$, hyaluronidase $(100 \mu \mathrm{g} / \mathrm{mL})$ and DNase $\mathrm{I}(100 \mu \mathrm{g} / \mathrm{mL})$ at $37^{\circ} \mathrm{C}$ for $25 \mathrm{~min}$ with persistent agitation. Digested cells were passed through a $40-\mu \mathrm{m}$ nylon mesh and collected by centrifugation at $500 \mathrm{~g}$ for $10 \mathrm{~min}$, followed by red blood cell (RBC) lysis. One hundred microliters of cell suspension $\left(2.0 \times 10^{7}\right.$ cells $\left./ \mathrm{mL}\right)$ were used for flow cytometry detection. For the analysis of $\mathrm{T}$ cell subpopulations, cells were stained with the following antibody cocktails: Alexa Fluor ${ }^{\circledR} 700$-conjugated antibody to CD45 (Clone: 30-F11, dilution: 1:200), FITC-conjugated antibody to CD3 (Clone: 17A2, dilution: 1:400), BV650-conjugated antibody to CD8 (Clone: 53-6.7, dilution: 1:200), PE/Dazzle 594-conjugated antibody to CD4 (Clone: GK1.5, 
dilution: 1:200), and PE-conjugated antibody to CD25 (Clone: 3C7, dilution: 1:100). BV, brilliant violet; FITC, fluorescein isothiocyanate; PE, phycoerythrin.

For intracellular cytokine staining, $2.0 \times 10^{6}$ tumor-infiltrating lymphocytes were seeded into 6-well plates in RPMI-1640 medium containing 10\% FBS and supplemented with eBioscience ${ }^{\text {Tx }}$ Cell Stimulation Cocktail (plus protein transport inhibitors). After stimulation, cells were stained for surface markers; fixed and permeabilized with Intracellular Staining Permeabilization Wash Buffer (BioLegend); followed by staining with PerCP-Cy5.5-conjugated antibody to IL2 (Clone: JES6-5H4, dilution: 1:200), PE-conjugated antibody to IFN- $\gamma$ (Clone: XMG1.2, dilution: 1:200) and Alexa Fluor ${ }^{\circledR}$ 647-conjugated antibody to Granzyme B (Clone: GB11, dilution: 1:200). All samples were acquired on a BD LSRFortessa ${ }^{\text {tx }}$ flow cytometer, and Data were analyzed using FlowJo v10 (Tree Star).

Statistics and reproducibility. All experiments have been reproduced at least two times, and all attempts at replication were successful with self-consistent results. All results are presented as means \pm standard deviation (s.d.), and differences with $P<0.05$ were considered significant. One-way and two-way analyses of variance (ANOVA) with a Tukey post-hoc test was used for multiple comparisons, and Student's $t$-test was used for two-group comparisons. Survival curves were analyzed using the log-rank (Mantel-Cox) test. Significance levels were defined as ns (not significant, $P>0.05$ ), ${ }^{*} P<0.05$, ${ }^{*} P P<0.01$, $* * * P<0.001$ and $* * * * P<0.0001$, $P$ values were calculated by GraphPad Prism 7 (GraphPad Software, Inc.) and marked on the figures.

Reporting summary. Further information on research design is available in the Nature Research Reporting Summary linked to this article.

\section{Data availability}

All data are available within the Article, Supplementary Information, or available from the authors upon request. Source data are provided with this paper.

Received: 30 January 2020; Accepted: 8 January 2021; Published online: 01 March 2021

\section{References}

1. Weiner, L. M., Surana, R. \& Wang, S. Monoclonal antibodies: versatile platforms for cancer immunotherapy. Nat. Rev. Immunol. 10, 317-327 (2010).

2. Kaplon, H. \& Reichert, J. M. Antibodies to watch in 2019. mAbs 11, 219-238 (2019).

3. Mayes, P. A., Hance, K. W. \& Hoos, A. The promise and challenges of immune agonist antibody development in cancer. Nat. Rev. Drug Discov. 17, 509-527 (2018).

4. Topalian, S. L., Drake, C. G. \& Pardoll, D. M. Immune checkpoint blockade: a common denominator approach to cancer therapy. Cancer Cell 27, 450-461 (2015).

5. Demaria, O. et al. Harnessing innate immunity in cancer therapy. Nature 574, 45-56 (2019).

6. Guillerey, C., Huntington, N. D. \& Smyth, M. J. Targeting natural killer cells in cancer immunotherapy. Nat. Immunol. 17, 1025-1036 (2016).

7. DeNardo, D. G. \& Ruffell, B. Macrophages as regulators of tumor immunity and immunotherapy. Nat. Rev. Immunol. 19, 369-382 (2019).

8. Velasquez, M. P., Bonifant, C. L. \& Gottschalk, S. Redirecting T cells to hematological malignancies with bispecific antibodies. Blood 131, 30-38 (2018).

9. Slaney, C. Y., Wang, P., Darcy, P. K. \& Kershaw, M. H. CARs versus BiTEs: a comparison between $\mathrm{T}$ cell-redirection strategies for cancer treatment. Cancer Discov. 8, 1-11 (2018).

10. Sadelain, M., Riviere, I. \& Riddell, S. Therapeutic T cell engineering. Nature 545, 423-431 (2017).

11. Labanieh, L., Majzner, R. G. \& Mackall, C. L. Programming CAR-T cells to kill cancer. Nat. Biomed. Eng. 2, 377-391 (2018).

12. Fry, T. J. et al. CD22-targeted CAR T cells induce remission in B-ALL that is naive or resistant to CD19-targeted CAR immunotherapy. Nat. Med. 24, 20-28 (2018).

13. Labrijn, A. F., Janmaat, M. L., Reichert, J. M. \& Parren, P. Bispecific antibodies: a mechanistic review of the pipeline. Nat. Rev. Drug Discov. 18, 585-608 (2019).

14. Baeuerle, P. A. \& Reinhardt, C. Bispecific T-cell engaging antibodies for cancer therapy. Cancer Res. 69, 4941-4944 (2009).

15. Ishiguro, T. et al. An anti-glypican 3/CD3 bispecific T cell-redirecting antibody for treatment of solid tumors. Sci. Transl. Med. 9, eaal4291 (2017).

16. Strohl, W. R. \& Naso, M. Bispecific T-cell redirection versus chimeric antigen receptor (CAR)-T cells as approaches to kill cancer cells. Antibodies 8, 1-68 (2019).
17. Clynes, R. A. \& Desjarlais, J. R. Redirected T cell cytotoxicity in cancer therapy. Annu. Rev. Med. 70, 437-450 (2019).

18. John, L. B. et al. Anti-PD-1 antibody therapy potently enhances the eradication of established tumors by gene-modified T cells. Clin. Cancer Res. 19, 5636-5646 (2013).

19. Krupka, C. et al. Blockade of the PD-1/PD-L1 axis augments lysis of AML cells by the CD33/CD3 BiTE antibody construct AMG 330: reversing a T-cellinduced immune escape mechanism. Leukemia 30, 484-491 (2016).

20. Khalil, D. N., Smith, E. L., Brentjens, R. J. \& Wolchok, J. D. The future of cancer treatment: immunomodulation, CARs and combination immunotherapy. Nat. Rev. Clin. Oncol. 13, 273-290 (2016).

21. Yuan, H. et al. Multivalent bi-specific nanobioconjugate engager for targeted cancer immunotherapy. Nat. Nanotech. 12, 763-769 (2017).

22. Mi, Y. et al. A dual immunotherapy nanoparticle improves T-cell activation and cancer immunotherapy. Adv. Mater. 30, 1706098 (2018).

23. Shen, M., Rusling, J. \& Dixit, C. K. Site-selective orientated immobilization of antibodies and conjugates for immunodiagnostics development. Methods 116, 95-111 (2017).

24. $\mathrm{Xu}, \mathrm{Y}$. et al. Structure, heterogeneity and developability assessment of therapeutic antibodies. $m A b s$ 11, 239-264 (2019).

25. Schroeder, H. W. Jr. \& Cavacini, L. Structure and function of immunoglobulins. J. Allergy Clin. Immunol. 125, S41-S52 (2010).

26. Leavy, O. Therapeutic antibodies: past, present and future. Nat. Rev. Immunol. 10, 297 (2010).

27. Kumar, S., Aaron, J. \& Sokolov, K. Directional conjugation of antibodies to nanoparticles for synthesis of multiplexed optical contrast agents with both delivery and targeting moieties. Nat. Protoc. 3, 314-320 (2008).

28. Reth, M. Matching cellular dimensions with molecular sizes. Nat. Immunol. 14, 765-767 (2013).

29. Polo, E. et al. Tips for the functionalization of nanoparticles with antibodies Methods Mol. Biol. 1051, 149-163 (2013).

30. Puertas, S. et al. Taking advantage of unspecific interactions to produce highly active magnetic nanoparticle-antibody conjugates. ACS Nano 5, 4521-4528 (2011).

31. Bhat, P., Leggatt, G., Waterhouse, N. \& Frazer, I. H. Interferon-gamma derived from cytotoxic lymphocytes directly enhances their motility and cytotoxicity. Cell Death Dis. 8, e2836 (2017).

32. Voskoboinik, I., Whisstock, J. C. \& Trapani, J. A. Perforin and granzymes: function, dysfunction and human pathology. Nat. Rev. Immunol. 15, 388-400 (2015).

33. Sagiv-Barfi, I. et al. Therapeutic antitumor immunity by checkpoint blockade is enhanced by ibrutinib, an inhibitor of both BTK and ITK. Proc. Natl Acad. Sci. USA 112, E966-E972 (2015).

34. Morvan, M. G. \& Lanier, L. L. NK cells and cancer: you can teach innate cells new tricks. Nat. Rev. Cancer 16, 7-19 (2016).

35. Zhang, Q. et al. Blockade of the checkpoint receptor TIGIT prevents NK cell exhaustion and elicits potent anti-tumor immunity. Nat. Immunol. 19 723-732 (2018)

36. Pathria, P., Louis, T. L. \& Varner, J. A. Targeting tumor-associated macrophages in cancer. Trends Immunol. 40, 310-327 (2019).

37. Weiskopf, K. et al. CD47-blocking immunotherapies stimulate macrophagemediated destruction of small-cell lung cancer. J. Clin. Invest. 126, 2610-2620 (2016).

38. Ries, C. H. et al. Targeting tumor-associated macrophages with anti-CSF-1R antibody reveals a strategy for cancer therapy. Cancer Cell 25, 846-859 (2014).

39. Havel, J. J., Chowell, D. \& Chan, T. A. The evolving landscape of biomarkers for checkpoint inhibitor immunotherapy. Nat. Rev. Cancer 19, 133-150 (2019).

40. Bi, J. \& Tian, Z. NK cell dysfunction and checkpoint immunotherapy. Front Immunol. 10, 1999 (2019)

41. Vivier, E. et al. Innate or adaptive immunity? The example of natural killer cells. Science 331, 44-49 (2011).

42. Weiskopf, K. \& Weissman, I. L. Macrophages are critical effectors of antibody therapies for cancer. $m A b s$ 7, 303-310 (2015).

43. Jenkins, R. W., Barbie, D. A. \& Flaherty, K. T. Mechanisms of resistance to immune checkpoint inhibitors. Br. J. Cancer 118, 9-16 (2018).

44. Patel, S. A. \& Minn, A. J. Combination cancer therapy with immune checkpoint blockade: mechanisms and strategies. Immunity 48, 417-433 (2018).

45. $\mathrm{Hu}, \mathrm{Q}$. et al. Conjugation of haematopoietic stem cells and platelets decorated with anti-PD-1 antibodies augments anti-leukaemia efficacy. Nat. Biomed. Eng. 2, 831-840 (2018)

46. Wang, D. et al. Engineering nanoparticles to locally activate T cells in the tumor microenvironment. Sci Immunol. 4, pii: eaau6584 (2019).

47. Ishihara, J. et al. Targeted antibody and cytokine cancer immunotherapies through collagen affinity. Sci. Transl. Med. 11, pii: eaau3259 (2019).

48. Brinkmann, U. \& Kontermann, R. E. The making of bispecific antibodies. $m A b s$ 9, 182-212 (2017). 
49. Kosmides, A. K., Sidhom, J. W., Fraser, A., Bessell, C. A. \& Schneck, J. P. Dual targeting nanoparticle stimulates the immune system to inhibit tumor growth. ACS Nano 11, 5417-5429 (2017).

50. Wu, L. et al. Trispecific antibodies enhance the therapeutic efficacy of tumordirected T cells through T cell receptor co-stimulation. Nat. Cancer 1, 86-98 (2020).

\section{Acknowledgements}

This work was financially supported by the National Key R\&D Program of China (2017YFA0205600), the National Natural Science Foundation of China (51633008, 32071380, and 31800831), the Science and Technology Program of Guangzhou (007306355061), the Program for Guangdong Introducing Innovative and Entrepreneurial Teams (2017ZT07S054), Guangdong Provincial Pearl River Talents Program (2017GC010713), the Outstanding Scholar Program of Bioland Laboratory (Guangzhou Regenerative Medicine and Health Guangdong Laboratory) (2018GZR110102001). We thank Professor Z.X. Lian from Chronic Disease Laboratory, South China University of Technology, for analyzing flow cytometric data.

\section{Author contributions}

C.-T.J. and K.-G.C. performed the experiments and analyzed the data. A.L, H.H., Y.-N.F., Q.-N.Y. aided C.-T.J. and K.-G.C. in the animal experiments. D.-K.Z. constructed B16F10-mCherry, B16-F10-OVA cells, and PDL1-KO-B16-F10 cells. H.-B.Z., J.-Z.D. and X.-Z.Y., contributed to the characterization of imNA. C.-F.X. and M.-H.X. provided valuable suggestions in the experimental design. J.W. and S.S. conceived the project, designed the experiments, analyzed the data, and wrote the manuscript. All the authors discussed the results and assisted in the preparation of the manuscript.

\section{Competing interests}

The authors declare no competing interests.

\section{Additional information}

Supplementary information The online version contains supplementary material available at https://doi.org/10.1038/s41467-021-21497-6.

Correspondence and requests for materials should be addressed to S.S. or J.W.

Peer review information Nature Communications thanks Betty Kim and Walter Storkus for their contribution to the peer review of this work. Peer review reports are available.

Reprints and permission information is available at http://www.nature.com/reprints

Publisher's note Springer Nature remains neutral with regard to jurisdictional claims in published maps and institutional affiliations.

(c) (i) Open Access This article is licensed under a Creative Commons Attribution 4.0 International License, which permits use, sharing, adaptation, distribution and reproduction in any medium or format, as long as you give appropriate credit to the original author(s) and the source, provide a link to the Creative Commons license, and indicate if changes were made. The images or other third party material in this article are included in the article's Creative Commons license, unless indicated otherwise in a credit line to the material. If material is not included in the article's Creative Commons license and your intended use is not permitted by statutory regulation or exceeds the permitted use, you will need to obtain permission directly from the copyright holder. To view a copy of this license, visit http://creativecommons.org/ licenses/by/4.0/.

(C) The Author(s) 2021 